\title{
The interaction of glueball and heavy-light flavoured meson in holographic QCD
}

\author{
Si-wen $\mathbf{L i}^{1, \mathrm{a}}$ \\ ${ }^{1}$ Department of Physics, School of Science, Dalian Maritime University, Dalian 116026, China
}

Received: 17 December 2018 / Accepted: 10 September 2020 / Published online: 22 September 2020

(C) The Author(s) 2020

\begin{abstract}
We construct the D4/D8 brane configuration in the Witten-Sakai-Sugimoto model by introducing a pair of heavy flavour brane with a heavy-light open string. The multiplets created by the heavy-light string acquire mass due to the finite separation of the heavy and light flavour branes thus they could be identified as the heavy-light meson fields in this model. On the other hand the glueball field is identified as the gravitational fluctuations carried by the close string in the bulk, so this model is able to describe the interaction of glueball and heavy-light meson through the openclose string interaction in gauge-gravity duality. We explicitly derive the effective action for the various glueballs and heavy-light mesons then numerically evaluate the associated lowest coupling constants. Afterwards the decay/production widths of various glueballs involving the lowest heavy-light meson, which is identified as $D^{0}$ meson, are calculated by using our effective action which might be remarkable to theoretically study the charm oscillation e.g. $D^{0}-\bar{D}^{0}, B_{s}-\bar{B}_{s}$ decay/production in hadron physics. This work extends the previous investigations of glueball in holographic QCD and it is also a further prediction of glueball-meson interaction.
\end{abstract}

\section{Contents}

1 Introduction ................. 1

2 The dynamics of glueball ... . . . . . . . 3

2.1 The geometry in supergravity . . . . . . . . 3

2.2 Gravitational fluctuations as the glueball field in holography ............. 4

3 The flavours and mesons . . . . . . . . . . . 5

3.1 The light flavour and meson . . . . . . . . 5

3.2 The heavy flavour and heavy-light flavoured meson . . . . . . . . . . . . 6

4 The interactions of glueball and heavy-light flavoured mesons . . . . . . . . . . . . 8 4.1 Involving the exotic scalar glueball $\ldots \ldots .9$

a e-mail: siwenli@dlmu.edu.cn (corresponding author)
4.2 Involving the dilatonic scalar glueball . . . . 10

4.3 Involving the tensor glueball . . . . . . . . . 11

5 Interaction of glueball and the lowest heavy-light meson in holography . . . . . . . . . . . . 11

5.1 The mode eigenfunctions and the choice of parameters . . . . . . . . . . 11

5.2 Glueball decay into the lowest scalar $D^{0} \bar{D}^{0}$ meson 12 6 Summary and discussion . . . . . . . . . . . . . . 14 Appendix A: The 10d equations of motion in the bulk supergravity . . . . . . . . . . . 15

Appendix B: The action of the D-brane with non-

Abelian excitation . . . . . . . . . . 15

Appendix C: The interactions of glueball and $\pi, \rho$ mesons 16

C1: The exotic scalar glueball . . . . . . . . . 16

C2: The dilatonic and tensor glueball $\ldots \ldots 16$ Appendix D: The renormalization of the quadric D8-

brane action . . . . . . . . . . 17

References . . . . . . . . . . . . . 17

\section{Introduction}

In nuclear physics, quantum chromodynamics $(\mathrm{QCD})$ as the underlying theory of the strong interaction allows to form bound states of pure gauge bosons due to its nonabelian nature. These bound states are named as "glueball"s [1-3] which are usually denoted by a range of quantum numbers $J^{P C}$ where $J, P, C$ represents total spin, parity and charge conjugation respectively. For instance, the lowest glueball takes the quantum numbers of the vacuum as $J^{P C}=0^{++}$. In Yang-Mills theory, glueball is believed as the only possible composite particle states and its spectrum has been detailedly studied by using lattice method [4-6]. While the evident existence of the glueball is expected, their experimental identification in the hadron spectra is challenging. This difficulty is mostly due to the mixing of glueball with $q \bar{q}$ (quark and anti-quark) states which have the same quantum numbers 
of the glueball in the presence of quarks. Nonetheless the mass of the lightest scalar glueball is predicted to be around 1600-1800 MeV by the simulations of lattice QCD [4,7]. However the coupling and decay/production widths of glueball have not been explicitly provided by the current status of lattice QCD since lattice theory including dynamical quarks expressed with real-time quantities would become extremely complexed.

Additional to the lattice gauge theory, an alternatively different approach to investigate strongly coupled quantum field theory has been proposed in [8,9] which is the famous anti-de Sitter/conformal field theory (AdS/CFT) correspondence, or more generally, the gauge-gravity (string) duality. In the AdS/CFT correspondence, the correlation functions of gauge invariant operators with the limitation of large $N_{c}$ (colour number) and large 't Hooft coupling $\lambda$ are mapped to the perturbations of the geometrical backgrounds in classical supergravity. So the correlation functions of strongly coupled quantum field theory could be calculated by the weakly coupled gravity theory in which the perturbation method is valid [10]. Significantly, a top-down construction for QCD based on $N_{c}$ D4-branes compactified on a circle in type-IIA string theory was proposed by Witten in 1998 [11] in which both supersymmetry and conformal symmetry are broken below a Kaluza-Klein mass scale $M_{K}$, so that the dual field theory is four-dimensional pure Yang-Mills theory in the large- $N_{c}$ limit. By embedding $N_{f}$ pairs of coincident D8- and anti D8branes (D8/D8-brane) as flavours into this D4-brane configuration, quarks in the fundamental representation of flavour and colour have been introduced into this model by Sakai and Sugimoto in 2004 [12,13]. Hence this model, named as the Witten-Sakai-Sugimoto (WSS) model, becomes remarkably successful since it almost contains all necessary ingredients of QCD and it is able to reproduce various fundamental features of low-energy nuclear physics with few parameters [14-21].

Particularly the glueball field in this model is identified as the gravitational perturbations in the bulk supergravity while meson and baryon states are created by the open strings on the flavoured D8/ $\overline{\mathrm{D} 8}$-brane and "baryonic D4-branes". ${ }^{1}$ So this model naturally includes the interaction of glueball and hadron which is nothing but the open-close string interaction in the viewpoint of string theory. Specifically the mass spectrum of glueball could be obtained by evaluating the eigen equation of the bulk graviton and the glueball-hadron interaction would arise in the Dirac-Born-Infield (DBI) action of the D-brane once the gravitational perturbation is involved [23-27].

\footnotetext{
1 We use "baryonic D4-brane" to refer to the D4-branes wrapped on $S^{4}$ which create the baryon states in this model. The details could be reviewed in [20,22].
}

In this paper, we will focus on the glueball-meson interaction by including the heavy flavour in order to extend the investigation in $[25,26]$ with this model. Since the fundamental quarks in this model are created by the open string with one end attached to the D4-brane and the other end to the D8/D8-brane which has zero length, it implies the fundamental quarks are massless. Thus the meson states consisted of these massless quarks are usually identified as the light flavour mesons, such as $\pi, \rho$ meson. To involve the heavy flavour, we are going to employ the mechanism proposed in [28,29], that is to introduce another pair of flavoured D8/ $\overline{\mathrm{D} 8}$-brane (as heavy flavour branes) separated from the other coincident D8/D8-brane (as light flavour branes) with a open string (as heavy-light string) stretched between them as illustrated in Fig. 2. Accordingly the multiplets created by the heavy-light string acquire mass due to the finite separation of the heavy and light flavour branes. So the model is able to include the heavy-light flavoured mesons with massive quarks in this approach and the mechanism is recognized as the "Higgs mechanism" in string theory [30,31]. Then the action for the interaction of glueball and heavy-light flavoured mesons could be derived by combining the gravitational perturbations in the bulk with the heavy-light meson fields on the flavour branes. As the most simple example, we finally evaluate the decay/production rates of glueball involving the lowest heavy-light mesons by using our effective action which might be remarkable to study charm oscillation as an effective theory e.g. $D^{0}-\bar{D}^{0}, B_{s}-\bar{B}_{s}$ decay/production in $[32,33]$ and the glueball decay/production involving the heavy-light mesons in hadron physics. Our results are also in agreement with the previous works in $[25,26,34]$.

The organization of this manuscript is as follows. In Sect. 2, we review the relation of $11 \mathrm{~d}$ M-theory and type IIA string theory by their corresponding supergravity. We also give the dynamics of the glueball from these supergravity systems in holography. In section 3, we briefly review the setup of the flavours in the WSS model and discuss how to involve the heavy flavour and heavy-light mesons. In Sect. 4, we detailedly derive the effective action for the interaction of various glueball and heavy-light meson in holography. In Sect. 5, we identify the lowest heavy-light meson in the model as the lowest D-meson through some experimental data and we use our effective action to compute the decay/production width of the glueball involving $D^{0}$ meson. The final section is the summary and our discussion about this work. At the end of this manuscript, we collect some essential discussion about the supergravity, the dynamics of D-brane, the supersymmetry, the glueball-light meson interaction and the holographic renormalization of this model in Appendix A, $\mathrm{B}, \mathrm{C}, \mathrm{D}$ respectively which are also useful to this work. 


\section{The dynamics of glueball}

\subsection{The geometry in supergravity}

The WSS model is based on the $\mathrm{AdS}_{7} / \mathrm{CFT}_{6}$ correspondence obtained by coincident $N_{c}$ M5-branes in 11-dimensional (11d) M-theory and the dual field theory on the M5-brane is a 6-dimensional $(0,2)$ superconformal field theory. The effective supergravity description provides a $11 \mathrm{~d}$ geometrical near-horizon solution which takes the topology of $A d S_{7} \times S^{4}$. Let us denote the extended directions of the M5-branes by $\left\{X^{\mu}, X^{4}, X^{11}\right\}$ with indices $\mu, v=0,1,2,3$, so the $11 \mathrm{~d}$ non-extremal black brane solution is given as [30], ${ }^{2}$

$$
\begin{aligned}
d s_{(11 \mathrm{~d}-\text { black })}^{2}= & \frac{r^{2}}{L^{2}}\left[f(r)\left(d X^{0}\right)^{2}\right. \\
& \left.+\delta_{i j} d X^{i} d X^{j}+\left(d X^{4}\right)^{2}+\left(d X^{11}\right)^{2}\right] \\
& +\frac{L^{2}}{r^{2}} \frac{d r^{2}}{f(r)}+\frac{L^{2}}{4} d \Omega_{4}^{2}, \\
f(r)= & 1-\frac{r_{K K}^{6}}{r^{6}},
\end{aligned}
$$

where $\Omega_{4}, L$ denotes the volume element on $S^{4}$ and the curvature radius of the $\mathrm{AdS}_{7}$ respectively. The indices $i, j$ run from 1 to 3 and $r$ represents the radius coordinate of the transverse directions. Note that this background also contains a non-vanished Ramond-Ramond strength $F_{4}$ with $N_{c}$ units of flux on $S^{4}$. As the 11d M-theory compactified on a cycle is equivalent to 10d type-IIA string theory, one can obtain nonconformal D4-branes by the dimensional reduction which preserves circle with

$$
X^{11} \sim X^{11}+2 \pi R_{11}, R_{11}=g_{s} l_{s}, l_{s}^{2}=\alpha^{\prime} .
$$

Afterwards, the dual field theory becomes a 5d super YangMills theory on the D4-branes.

In order to obtain a pure Yang-Mills or QCD-like theory, a further compactification with a double Wick-rotation was proposed by Witten based on the above holographic duality. Accordingly the metric (2.1) with the double Wick-rotation $X^{0} \rightarrow-i X^{4}, X^{4} \rightarrow-i X^{0}$ corresponds to a bubble configuration of D4-brane which is given as [11,12],

$$
\begin{aligned}
d s_{(11 \mathrm{~d}-\text { bubble })}^{2}= & \frac{r^{2}}{L^{2}}\left[\eta_{\mu \nu} d X^{\mu} d X^{\nu}+f(r)\left(d X^{4}\right)^{2}+\left(d X^{11}\right)^{2}\right] \\
& +\frac{L^{2}}{r^{2}} \frac{d r^{2}}{f(r)}+\frac{L^{2}}{4} d \Omega_{4}^{2},
\end{aligned}
$$

\footnotetext{
$\overline{2}$ The equations of motion for the bulk fields can also been found in the Appendix A.
}

so that the dual field theory exhibits confinements in this geometry. The supersymmetry of the D4-branes is broken down by compactifying $X^{4}$ on another circle, hence the fermionic gluinos become massive at tree level by imposing the antiperiodic boundary conditions and the adjoint scalars also acquire masses through its loop corrections. Therefore the gauge bosons are the only degrees of freedom in the limit of large Kaluza-Klein (KK) mass scale. Employing the relations of the dimensional reduction, the $10 \mathrm{~d}$ metric could be written as $[25,26,35]$,

$d s_{(11 \mathrm{~d})}^{2}=e^{-2 \Phi / 3} d s_{(10 \mathrm{~d})}^{2}+e^{4 \Phi / 3}\left(d X^{11}+A_{M} d X^{M}\right)^{2}$

where $M=0,1 \ldots 9$ and $\Phi=\frac{3}{2} \ln \left(\frac{r}{L}\right)$ is the $10 \mathrm{~d}$ dilaton field. Note that according to the above discussion we have $A_{m}=0$ and a periodic condition for $X^{4}$,

$X^{4} \sim X^{4}+2 \pi \delta X^{4}, \delta X^{4}=\frac{1}{M_{K K}}=\frac{L^{2}}{3 r_{K K}}$.

The relation of $r_{K K}$ and $M_{K K}$ can be determined by eliminating the conical singularity at $r=r_{K K}$. Using alternative radial coordinates $U \in\left[U_{K K},+\infty\right)$ and $Z \in[-\infty,+\infty)$ defined as

$L=2 R, \quad U=\frac{r^{2}}{2 L}, \quad K(Z)=1+Z^{2}=\frac{r^{6}}{r_{K K}^{6}}=\frac{U^{3}}{U_{K K^{3}}}$,

the $10 \mathrm{~d}$ metric in (2.4) can be explicitly written as,

$$
\begin{aligned}
d s_{(10 \mathrm{~d})}^{2}= & \left(\frac{U}{R}\right)^{3 / 2}\left[\eta_{\mu \nu} d X^{\mu} d X^{\nu}+f(U)\left(d X^{4}\right)^{2}\right] \\
& +\left(\frac{R}{U}\right)^{3 / 2}\left[\frac{d U^{2}}{f(U)}+U^{2} d \Omega_{4}^{2}\right], \\
f(U) & =1-\frac{U_{K K}^{3}}{U^{3}}, \quad e^{\Phi}=\left(\frac{U}{R}\right)^{3 / 4}, \\
F_{4} & =d C_{3}=\frac{2 \pi N_{c}}{V_{4}} \epsilon_{4},
\end{aligned}
$$

where $\epsilon_{4}$ denotes a unit volume element on $S^{4}$ and $F_{4}$ is the field strength of the Ramond-Ramond form $C_{3}$. Note that the metric in (2.7) corresponds to $\mathrm{D} 4$ bubble configuration which is obtained by a double Wick-rotation from the black brane solution. And the 11th direction $X^{11}$ becomes vanished in the large $N_{c}$ limit. In terms of QCD variables we additionally have the following relations,

$$
\lambda=g_{\mathrm{YM}}^{2} N_{c}, \quad g_{\mathrm{YM}}^{2}=2 \pi g_{s} l_{s} M_{K K}, \quad R^{3}=\pi g_{s} N_{c} l_{s}^{3},
$$


where $\lambda, g_{\mathrm{YM}}, g_{s}, l_{s}$ respectively represents the 't Hooft coupling constant, Yang-Mills coupling constant, string coupling constant and the length of string.

\subsection{Gravitational fluctuations as the glueball field in holography}

Since glueball is the gauge invariant composite state in the Yang-Mills theory, they holographically corresponds to the gravitational perturbations of the near-horizon geometry in the supergravity description [23-27]. Such metric fluctuations are sourced by the operators in the dual field theory i.e. the 5d compactified Yang-Mills theory. Hence let us introduce the gravitational perturbations to the background (2.3) by rewriting the $11 \mathrm{~d}$ metric as $G_{M N} \rightarrow G_{M N}^{(0)}+\delta G_{M N}$ where $G_{M N}^{(0)}$ refers to the metric in (2.3). And it would be convenient to integrate over the $S^{4}$ in the $11 \mathrm{~d}$ supergravity action since $S^{4}$ is not very necessary in the following discussion. So we can obtain the kinetic action for glueball by imposing the metric (2.3) into the $11 \mathrm{~d}$ supergravity action and it leads to,

$$
\begin{aligned}
S_{11 \mathrm{D}} & =\frac{1}{2 \kappa_{11}^{2}}\left(\frac{L}{2}\right)^{4} \Omega_{4} \int d^{7} x \sqrt{-\operatorname{det} G}\left(\mathcal{R}_{11 \mathrm{D}}+\frac{30}{L^{2}}\right) \\
& =\frac{1}{2 \kappa_{11}^{2}}\left(\frac{L}{2}\right)^{4} \Omega_{4} \mathcal{C}_{E, D, T} S_{G}
\end{aligned}
$$

where $S_{G}$ denotes the kinetic term of the glueball and $\mathcal{C}_{E, D, T}$ is a constant determined by the various gravitational fluctuations. Note that the pre-factor in the above action has to be normalized as $\frac{1}{2 \kappa_{11}^{2}}\left(\frac{L}{2}\right)^{4} \Omega_{4} \mathcal{C}_{E, D, T}=1$ in which $\mathcal{C}_{E, D, T}$ is completely fixed. We will use this action to discuss the dynamics of the glueball with various gravitational perturbations in the the rest of the manuscript.

\subsubsection{The exotic scalar glueball}

The lowest exotic scalar glueball has quantum number $J^{C P}=0^{++}$which corresponds to the exotic polarizations of the bulk graviton. The $11 \mathrm{~d}$ components of $\delta G_{M N}$ are given as [26],

$$
\begin{aligned}
\delta G_{44}= & -\frac{r^{2}}{L^{2}} f(r) H_{E}(r) G_{E}(x) \\
\delta G_{\mu \nu}= & \frac{r^{2}}{L^{2}} H_{E}(r)\left[\frac{1}{4} \eta_{\mu \nu}\right. \\
& \left.-\left(\frac{1}{4}+\frac{3 r_{K K}^{6}}{5 r^{6}-2 r_{K K}^{6}}\right) \frac{\partial_{\mu} \partial_{\nu}}{M_{E}^{2}}\right] G_{E}(x), \\
\delta G_{11,11}= & \frac{r^{2}}{4 L^{2}} H_{E}(r) G_{E}(x),
\end{aligned}
$$

$$
\begin{aligned}
\delta G_{r r} & =-\frac{L^{2}}{r^{2}} \frac{1}{f(r)} \frac{3 r_{K K}^{6}}{5 r^{6}-2 r_{K K}^{6}} H_{E}(r) G_{E}(x), \\
\delta G_{r \mu} & =\frac{90 r^{7} r_{K K}^{6}}{M_{E}^{2} L^{2}\left(5 r^{6}-2 r_{K K}^{6}\right)^{2}} H_{E}(r) \partial_{\mu} G_{E}(x),
\end{aligned}
$$

where the eigenvalue equation for function $H_{E}(r)$ is,

$$
\begin{aligned}
& \frac{1}{r^{3}} \frac{d}{d r}\left[r\left(r^{6}-r_{K K}^{6}\right) \frac{d}{d r} H_{E}(r)\right] \\
& \quad+\left[\frac{432 r^{2} r_{K K}^{12}}{\left(5 r^{6}-2 r_{K K}^{6}\right)^{2}}+L^{4} M_{E}^{2}\right] H_{E}(r)=0 .
\end{aligned}
$$

Note that the above components in (2.10) involve asymptotics in the bulk as $\delta G_{44}=-4 \delta G_{11}=-4 \delta G_{22}=-4 \delta G_{33}=$ $-4 \delta G_{11,11}$ for $r \rightarrow \infty$. Imposing the metric (2.3) with fluctuations (2.10) and the eigenvalue equation for function $H_{E}(r)$ into action (2.9), we obtain the kinetic term of the exotic scalar glueball which is,

$S_{G_{E}(x)}=-\frac{1}{2} \int d^{4} x\left[\left(\partial_{\mu} G_{E}\right)^{2}+M_{E}^{2} G_{E}^{2}\right]$,

where

$\mathcal{C}_{E}=\int_{r_{K} K}^{\infty} d r \frac{r^{3}}{L^{3}} \frac{5}{8} H_{E}^{2}(r)$.

\subsubsection{The dilatonic and tensor glueball}

The scalar glueball of $0^{++}$mode corresponds to the fluctuations of the metric as [26],

$$
\begin{aligned}
\delta G_{11,11} & =-3 \frac{r^{2}}{L^{2}} H_{D}(r) G_{D}(x), \\
\delta G_{\mu \nu} & =\frac{r^{2}}{L^{2}} H_{D}(r)\left[\eta^{\mu \nu}-\frac{\partial^{\mu} \partial^{v}}{M_{D}^{2}}\right] G_{D}(x) .
\end{aligned}
$$

We refer to the upon mode as "dilatonic" since $\delta G_{11,11}$ reduces to the $10 \mathrm{~d}$ dilaton.

The tensor glueball with $J^{C P}=2^{++}$corresponds to the metric fluctuations which includes a transverse traceless polarization. A choice of the non-vanishing components of the graviton polarizations could be,

$\delta G_{\mu \nu}=-\frac{r^{2}}{L^{2}} H_{T}(r) T_{\mu \nu}(x)$,

where $T_{\mu \nu} \equiv \mathcal{T}_{\mu \nu} G_{T}(x) . \mathcal{T}_{\mu \nu}$ is a constant symmetric tensor normalized as $\mathcal{T}_{\mu \nu} \mathcal{T}^{\mu \nu}=1$ and satisfies the traceless condition $\eta^{\mu \nu} \mathcal{T}_{\mu \nu}=0$. The eigenvalue equation for functions $H_{D, T}(r)$ are given as, 
Table 1 The D-brane configurations: "-” denotes the world volume directions of the D-branes

\begin{tabular}{llllllllll}
\hline & 0 & 1 & 2 & 3 & $(4)$ & $5(U)$ & 6 & 7 & 8 \\
\hline$N_{c} \mathrm{D} 4$ & - & - & - & - & - & & & & \\
$N_{f} \mathrm{D} 8 / \overline{\mathrm{D} 8}$ & - & - & - & - & & - & - & - & - \\
\hline
\end{tabular}

$$
\begin{aligned}
& \frac{1}{r^{3}} \frac{d}{d r}\left[r\left(r^{6}-r_{K K}^{6}\right) \frac{d}{d r} H_{D, T}(r)\right] \\
& +L^{4} M_{D, T}^{2} H_{D, T}(r)=0 .
\end{aligned}
$$

Plugging metric (2.3) with fluctuations (2.14) and (2.15) into action (2.9) respectively leads to the dynamics of the dilatonic scalar and tensor glueball,

$$
\begin{aligned}
S_{G_{D}(x)} & =-\frac{1}{2} \int d^{4} x\left[\left(\partial_{\mu} G_{D}\right)^{2}+M_{D}^{2} G_{D}^{2}\right], \\
S_{T(x)} & =-\frac{1}{4} \int d^{4} x\left[T_{\mu \nu}\left(\partial^{2}-M_{T}^{2}\right) T^{\mu \nu}\right],
\end{aligned}
$$

where the corresponding normalization constant is,

$$
\begin{aligned}
& \mathcal{C}_{D}=6 \int_{r_{K K}}^{\infty} d r \frac{r^{3}}{L^{3}} H_{D}^{2}(r), \\
& \mathcal{C}_{T}=2 \int_{r_{K K}}^{\infty} d r \frac{r^{3}}{L^{3}} H_{T}^{2}(r) .
\end{aligned}
$$

\section{The flavours and mesons}

\subsection{The light flavour and meson}

There are also flavours in this model which are introduced by embedding a stack of $N_{f}$ probe D8- and anti-D8-branes (D8/D8-brane) at the antipodal position of the background geometry. The configurations of the various branes are illustrated in Table 1.

The $N_{f}$ flavoured fermions arise from the lowest modes of the 4-8 strings ${ }^{3}$ and $4-\overline{8}$ strings which belong to the fundamental representation of the colour group $U\left(N_{c}\right)$ and flavour group $U\left(N_{f}\right)$. In order to define the chirality of these fundamental fermions, a natural choice is to perform the GSO (Gliozzi-Scherk-Olive) projection [12,36]. Since the GSO projection for $4-8$ strings is opposite to the projection chosen for the $4-\overline{8}$ strings, these fundamental fermions could be interpreted as quarks with opposite chiralities in QCD. And the gauge symmetry introduced by $\mathrm{D} 8 / \overline{\mathrm{D} 8}$-branes can be

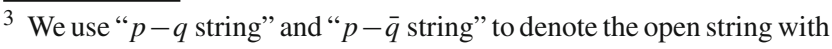
one end attached to the $\mathrm{D} p$-brane and the other end to the $\mathrm{D} q$-brane and $\overline{\mathrm{D} q}$-brane respectively. For example, " $4-\overline{8}$ string" refers to the open string with one end attached to the D4-brane and the other end to the $\overline{\mathrm{D}}$-brane.
}

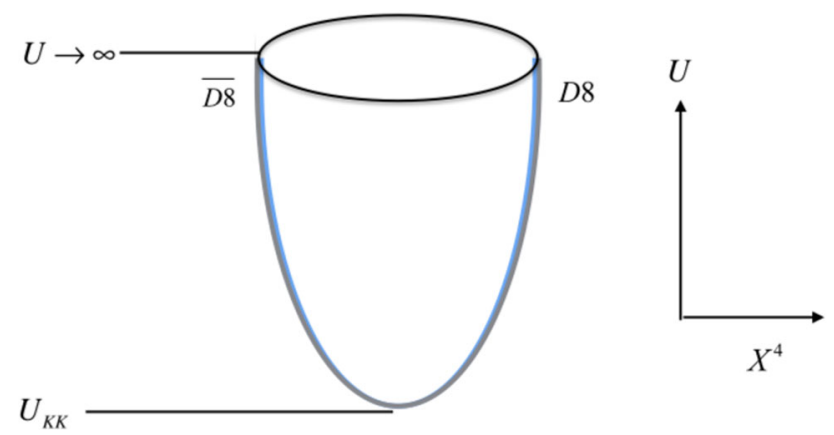

Fig. 1 The D-brane configuration in the $X^{4}-U$ plane where $X^{4}$ is compactified on $S^{1}$. The bubble background (cigar) is produced by $N_{c}$ D4-branes . The $N_{f}$ light flavour D8/D8-branes (L) living at antipodal position of the cigar are represented by the blue line. The D8/ $\overline{\mathrm{D} 8}$-branes are connected to each other at the bottom of the bulk $\left(U=U_{K K}\right)$

therefore denoted as $U\left(N_{f}\right)_{L} \times U\left(N_{f}\right)_{R}$, namely the chiral symmetry. Note that the $4-8 / \overline{8}$ strings has zero length in this D4/D8 configuration, the fundamental fermions are therefore massless which correspond accordingly to the light flavoured quarks in QCD. In this sense it motivates us to name these flavour branes as "light flavour branes". As shown in Table 1, the quarks and anti-quarks live on the separate position of $X^{4}$. However the global chiral symmetry $U\left(N_{f}\right)_{L} \times U\left(N_{f}\right)_{R}$ is broken spontaneously since the geometry of the D4 bubble configuration forces the $\mathrm{D} 8 / \overline{\mathrm{D} 8}$-brane to be connected at the bottom of the bulk as illustrated in Fig. 1.

Thus the induced metric on the flavour branes could be straightforward obtained by putting $\mathrm{D} 8 / \overline{\mathrm{D}} 8$-branes onshell i.e. imposing the condition $\frac{d x^{4}}{d U}=0$, then we have,

$$
\begin{aligned}
d s_{\mathrm{D} 8}^{2}= & g_{M N} d X^{M} d X^{N}=\left(\frac{U}{R}\right)^{3 / 2} \eta_{\mu \nu} d x^{\mu} d x^{\nu} \\
& +\left(\frac{R}{U}\right)^{3 / 2}\left[\frac{d U^{2}}{f(U)}+U^{2} d \Omega_{4}^{2}\right]
\end{aligned}
$$

where the indices $M, N$ run over the flavour brane. So the action of the joined light flavour branes describes the dynamics of $q \bar{q}$ mesons, which contains the light quarks only, through the flavoured gauge fields on the worldvolume of

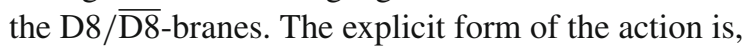

$S_{\mathrm{D} 8}=S_{\mathrm{DBI}}+S_{\mathrm{CS}}$, 
where $^{4}$

$$
\begin{aligned}
S_{\mathrm{DBI}}= & -T_{8} \operatorname{Tr} \int_{\mathrm{D} 8 / \overline{\mathrm{D} 8}} d^{9} x e^{-\Phi} \sqrt{-\operatorname{det}\left(g+2 \pi \alpha^{\prime} F\right)} \\
= & -T_{8} \operatorname{Tr} \int_{\mathrm{D} 8 / \overline{\mathrm{D} 8}} d^{9} x e^{-\Phi} \sqrt{-g} \\
& \times\left[1+\frac{1}{4}\left(2 \pi \alpha^{\prime}\right)^{2} g^{M N} g^{K L} F_{M K} F_{N L}+\mathcal{O}\left(F^{4}\right)\right], \\
S_{\mathrm{CS}}= & \mu_{8} \int_{\mathrm{D} 8 / \overline{\mathrm{D} 8}} C_{3} \wedge\left(2 \pi \alpha^{\prime}\right)^{3} \operatorname{Tr}(F \wedge F \wedge F) .
\end{aligned}
$$

Here $g$ and $\Phi$ is the induced metric and dilaton given in (2.7), (3.1), respectively. $F$ refers to the field strength of the flavoured gauge field $A_{M}$ on the D8-branes which is defined as $F_{M N}=\partial_{M} A_{N}-\partial_{N} A_{M}+\left[A_{M}, A_{N}\right]^{5}$. Note that in this model the non-zero components of the gauge field $A_{M}$ are only $\left\{A_{\mu}, A_{Z}\right\}$ which are functions dependent on the coordinates $\left\{x^{\mu}, Z\right\}$. Since the flavour branes are probes, we could obtain the following $5 \mathrm{~d}$ quadratic action after imposing the solution (3.1), (2.7)-(3.3) and integrating over $S^{4}$ which is,

$$
\begin{aligned}
S_{\mathrm{DBI}}^{\mathrm{YM}}= & -\kappa \operatorname{Tr} \int d^{4} x \int_{-\infty}^{+\infty} d Z\left[\frac{1}{2} K(Z)^{-1 / 3} \eta^{\mu \rho} \eta^{\nu \sigma} F_{\mu \nu} F_{\rho \sigma}\right. \\
& \left.+K(Z) M_{K K}^{2} \eta^{\mu \nu} F_{\mu Z} F_{\nu Z}\right]
\end{aligned}
$$

where

$$
\begin{aligned}
\kappa & =\frac{1}{3}\left(2 \pi \alpha^{\prime}\right)^{2} T_{8} g_{s}^{-1} \omega_{4} R^{9 / 2} U_{K K}^{1 / 2}=\frac{\lambda N_{c}}{216 \pi^{3}}, \\
\omega_{4} & =\frac{8 \pi^{2}}{3}, \quad M_{K K}^{2}=\frac{9 U_{K K}}{4 R^{3}} .
\end{aligned}
$$

The light flavour meson fields are identified as the expansion modes of $A_{M}$ by a complete sets $\left\{\psi_{n}\right\}$ in the model. Explicitly $A_{M}$ is assumed to be expanded as ${ }^{6}$,

$$
\begin{aligned}
A_{\mu}= & \sum_{n=1} B_{\mu}^{(n)}(x) \psi_{n}(Z), \\
A_{Z}= & \varphi^{(0)}(x) \psi_{0}^{\prime}(Z) \\
& +\sum_{n=1} m_{n}^{-1} \varphi^{(n)}(x) \psi_{n}^{\prime}(Z) .
\end{aligned}
$$

so the lowest mode of $A_{\mu}$ and $A_{Z}$ could be interpreted as the lightest vector $\rho$ meson and scalar $\pi$ meson. Therefore if we

\footnotetext{
${ }^{4}$ For $N_{f}$ D8-branes, all the actions in (3.3) should include a pre-factor $N_{f}$. Nonetheless we do not pick it up because a pre-factor does not affect any results.

${ }^{5}$ In this notation, the gauge field $A_{M}$ is anti-Hermitian which means $A_{M}^{\dagger}=-A_{M}$ and $F_{M N}^{\dagger}=-F_{M N}$.

${ }^{6}$ In (3.4) there is a residual gauge symmetry in the action. So it would be convenient to choose a gauge which keeps $A_{Z}$ nonzero for our purposes. The frequently chosen gauge which keeps $A_{Z}=0$ leads to a different parametrization of the Goldstone bosons in this model but physically equivalent situation.
}

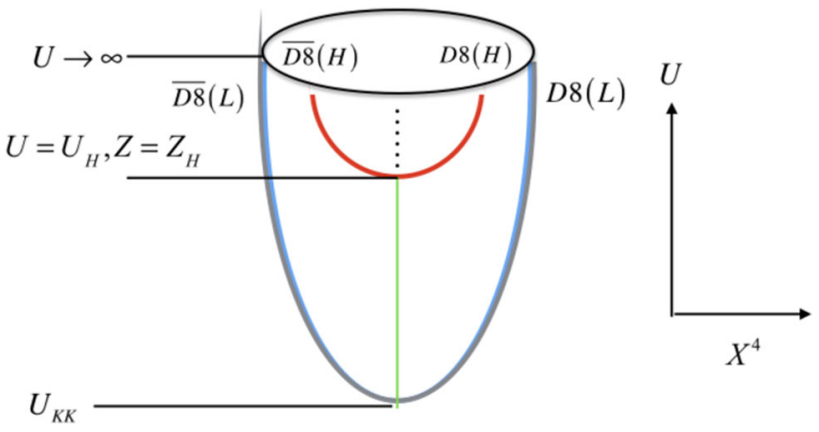

Fig. 2 The D-brane configuration involving the heavy flavour in the $X^{4}-U$ plane. Additional to the light flavour branes, a pair of heavy flavour $\mathrm{D} 8 / \overline{\mathrm{D} 8}$-brane $(\mathrm{H})$ (denoted by the red line) is introduced in to the D4/D8 configuration which is separated from the light flavour branes. The heavy flavour D8/D8 -branes are connected to each other at $U=U_{H}>U_{K K}$ and the massive multiplets are produced by the heavylight string (HL-string denoted by the green line) stretched between the light and heavy flavour branes which is denoted by the green line in this figure

identify $B_{\mu}^{(1)}(x)=\rho_{\mu}(x), \varphi^{(0)}(x)=U_{K} \pi(x)$ with the normalization,

$$
\begin{aligned}
& 2 \kappa \int_{-\infty}^{+\infty} d Z K(Z)^{-1 / 3}\left[\psi_{1}(Z)\right]^{2}=1, \\
& 2 \kappa\left(U_{K K} M_{K K}\right)^{2} \int_{-\infty}^{+\infty} d Z K(Z)\left[\psi_{0}^{\prime}(Z)\right]^{2}=1,
\end{aligned}
$$

the effective $4 \mathrm{~d}$ action of $\pi$ and $\rho$ meson denoted by $S_{\pi-\rho}^{\text {eff }}$ can be obtained from (3.4) whose form is ${ }^{7}$,

$$
\begin{aligned}
S_{\pi-\rho}^{\mathrm{eff}}= & -\operatorname{Tr} \int d^{4} x\left[\frac{1}{2}\left(\partial_{\mu} \pi\right)^{2}+\frac{1}{4} \partial_{[\mu} \rho_{\nu]} \partial^{[\mu} \rho^{\nu]}\right. \\
& \left.+\frac{1}{2} \lambda_{1} M_{K K}^{2} \rho_{\mu}^{2}+\cdots \text { (interactions) }\right] .
\end{aligned}
$$

The Chern-Simons (CS) term in (3.3) corresponds to the Wess-Zumino-Witten (WZW) term in chiral QCD. However we will not discuss its explicit form in this manuscript because it is independent on the metric thus does not include the interaction of meson and glueball.

\subsection{The heavy flavour and heavy-light flavoured meson}

The heavy flavour could be introduced into this model by considering an extra pair of flavoured D8/D8-brane which is separated from the other $N_{f}$ (light-flavoured) $\mathrm{D} 8 / \overline{\mathrm{D} 8}$ branes with an open string (the heavy-light string) stretched between them $[28,29,37,38]$ as illustrated in Fig. 2. According to string theory, the heavy-light (HL) string stretched between the separated branes creates additional multiplets which could be approximated near the worldvolume of the

\footnotetext{
7 The dynamics of $\varphi^{(1)}$ is absorbed by the $\rho$ meson through a gauge transformation $B_{\mu}^{(1)} \rightarrow B_{\mu}^{(1)}+m_{n}^{-1} \partial_{\mu} \varphi^{(1)}$.
} 
light flavour branes by local vector fields. Due to the finite separation of the heavy and light flavour branes, the HLstring has a non-zero length so that those multiplets acquire mass through the non-zero vacuum expectation value (VEV) of the HL-string. Hence the multiplets could be interpreted as the heavy-flavoured mesons with massive quarks. We note that this mechanism to acquire the massive fields is exactly the "Higgs mechanism" in string theory [30].

In order to include HL-multiple states in the actions (3.3), we need to replace the gauge fields on the light flavour branes by its matrix-valued formula according to string theory,

$$
A_{M} \rightarrow \mathbf{A}_{M}=\left(\begin{array}{cc}
A_{M} & \Phi_{M} \\
-\Phi_{M}^{\dagger} & 0
\end{array}\right)
$$

In our setup $\mathbf{A}_{M}$ is $\left(N_{f}+1\right) \times\left(N_{f}+1\right)$ matrix-valued 1form while $A_{M}$ is $N_{f} \times N_{f}$ valued 1-form. And $\Phi_{M}$ is $N_{f} \times$ 1 valued vector. Thus the field strength of $\mathbf{A}_{M}$ becomes a matrix-valued 2-form as,

$$
\begin{aligned}
F_{M N} \rightarrow \mathbf{F}_{M N} & \\
& =\left(\begin{array}{rc}
F_{M N}-\Phi_{[M} \Phi_{N]}^{\dagger} & \partial_{[M} \Phi_{N]}+A_{[M} \Phi_{N]} \\
-\partial_{[M} \Phi_{N]}^{\dagger}-\Phi_{[M}^{\dagger} A_{N]} & -\Phi_{[M}^{\dagger} \Phi_{N]}
\end{array}\right) .
\end{aligned}
$$

And we have assumed that the non-zero components of $\Phi_{M}$ are $\left\{\Phi_{\mu}, \Phi_{Z}\right\}$ which depends only on $\left\{x^{\mu}, Z\right\}$ in order to be compatible with $A_{M}$. Plugging (3.9), (3.10) into the quadratic action (3.4), we obtain the action as,

$$
\begin{aligned}
S_{\mathrm{DBI}}^{\mathrm{YM}}= & -\frac{1}{4}\left(2 \pi \alpha^{\prime}\right)^{2} T_{8} \operatorname{Tr} \int_{\mathrm{D} 8 / \overline{\mathrm{D} 8}} d^{9} x e^{-\Phi} \sqrt{-g} g^{M N} g^{K L} \mathbf{F}_{M K} \mathbf{F}_{N L} \\
= & -\kappa \operatorname{Tr} \int d^{4} x \int_{-\infty}^{+\infty} d Z\left[\frac{1}{2} K(Z)^{-1 / 3} \eta^{\mu \rho} \eta^{\nu \sigma} \mathbf{F}_{\mu \nu} \mathbf{F}_{\rho \sigma}\right. \\
& \left.+K(Z) M_{K K}^{2} \eta^{\mu \nu} \mathbf{F}_{\mu Z} \mathbf{F}_{\nu Z}\right] . \\
= & -\kappa \operatorname{Tr} \int d^{4} x \int_{-\infty}^{+\infty} d Z\left\{\frac { 1 } { 2 } K ( Z ) ^ { - 1 / 3 } \left[\left(F_{\mu \nu}-a_{\mu \nu}\right)\right.\right. \\
& \left.\times\left(F^{\mu \nu}-a^{\mu \nu}\right)-2 f_{\mu \nu}^{\dagger} f^{\mu \nu}+b_{\mu \nu} b^{\mu \nu}\right] \\
& +K(Z) M_{K K}^{2} \eta^{\mu \nu}\left[\left(F_{\mu Z}-a_{\mu Z}\right)\left(F_{\nu Z}-a_{\nu Z}\right)\right. \\
& \left.\left.-2 f_{\mu Z}^{\dagger} f_{\nu Z}+b_{\mu Z} b_{\nu Z}\right]\right\},
\end{aligned}
$$

where "Tr" refers to the "flavour trace" and

$$
\begin{aligned}
& f_{M N}^{\dagger}=-\partial_{[M} \Phi_{N]}^{\dagger}-\Phi_{[M}^{\dagger} A_{N]}, \quad b_{M N}=\Phi_{[M}^{\dagger} \Phi_{N]}, \\
& f_{M N}=\partial_{[M} \Phi_{N]}+A_{[M} \Phi_{N]}, \quad a_{M N}=\Phi_{[M} \Phi_{N]}^{\dagger} .
\end{aligned}
$$

Note that the DBI action has to involve the transverse modes of the brane since the HL-string with finite separation provides a non-zero VEV in our setup. For the reader's con- venience, we have summarized the essential derivations to obtain the quadratic action in (3.3) from the complete DBI action with non-Abelian excitation in the Appendix B. Then let us denote the only one transverse "coordinate" as $\Psi$ for D8-branes and its T-dualitized action is given as,

$$
\begin{aligned}
S_{\Psi}= & -\tilde{T}_{8} \operatorname{Tr} \int_{\mathrm{D} 8 / \overline{\mathrm{D} 8}} d^{9} x e^{-\Phi} \sqrt{-\operatorname{det} g} \\
& \times\left\{\frac{1}{2} D_{M} \Psi D^{M} \Psi+\frac{1}{4}[\Psi, \Psi]^{2}\right\},
\end{aligned}
$$

with $D_{M} \Psi=\partial_{M} \Psi+\left[\mathbf{A}_{M}, \Psi\right]$ and $\tilde{T}_{8}=\left(2 \pi \alpha^{\prime}\right)^{2} T_{8}$. By the extrema of the potential contribution or $[\Psi,[\Psi, \Psi]]=0$, we can define the moduli solution of $\Psi$ for $N_{f}$ light branes separated from one heavy brane with a finite VEV $v$ as [30, 31,37-39],

$$
\Psi=\left(\begin{array}{cc}
-\frac{v}{N_{f}} \mathbf{1}_{N_{f}} & 0 \\
0 & v
\end{array}\right) .
$$

By using the solution (3.15), the action (3.14) could be rewritten as,

$$
\begin{aligned}
S_{\Psi}= & -\tilde{T}_{8} v^{2} \frac{\left(N_{f}+1\right)^{2}}{N_{f}^{2}} \operatorname{Tr} \int d^{4} x \\
& \times \int_{-\infty}^{+\infty} d Z e^{-\Phi} \sqrt{-\operatorname{det} g} g^{M N} \Phi_{M}^{\dagger} \Phi_{N},
\end{aligned}
$$

We note here the total action of the light flavour branes involving the HL fields is collected as,

$S_{\mathrm{D} 8}=S_{\mathrm{DBI}}^{\mathrm{YM}}+S_{\Psi}+S_{\mathrm{CS}}$,

where $S_{\mathrm{CS}}$ takes the same formula as (3.3) but replacing $F \rightarrow$ F.

Along the discussion in the sector of light flavour, we further assume that the HL-multiplet $\Phi_{M}$ could be in general expanded as [28],

$\Phi_{\mu}=\epsilon_{\mu}(z) e^{i p \cdot x} \equiv \sum_{m} Q_{\mu, m}(x) \phi_{m}(Z)$,

$\Phi_{Z}=\epsilon(z) e^{i p \cdot x} \equiv U_{K K} \sum_{m} Q_{m}(x) \tilde{\phi}_{m}(Z)$.

By inserting (3.18) into the the equations of motion following from (3.17), for the transverse mode we can choose the decomposition as,

$\phi_{m}(Z) \neq 0, \quad \tilde{\phi}_{m}(Z) \equiv 0$, 
which leads to the action of the heavy-light vector meson field,

$$
\begin{aligned}
S_{\mathrm{HL}}= & -\operatorname{Tr} \int d^{4} x \sum_{n}\left[\frac{1}{4} \partial_{[\mu} Q_{\nu], n}^{\dagger} \partial^{[\mu} Q_{, n}^{\nu]}\right. \\
& \left.+\frac{1}{2}\left(\Lambda_{n} M_{K K}^{2}+M_{V, n}^{2}\right) Q_{\mu, n}^{\dagger} Q_{, n}^{\mu}\right],
\end{aligned}
$$

with the normalization condition

$4 \kappa \int_{-\infty}^{+\infty} d Z K(Z)^{-1 / 3} \phi_{m}(Z) \phi_{n}(Z)=\delta_{m n}$,

$4 \kappa \int_{-\infty}^{+\infty} d Z K(Z) \phi_{m}^{\prime}(Z) \phi_{n}^{\prime}(Z)=\Lambda_{n} \delta_{m n}$.

$M_{V, n}^{2}=v_{V}^{2} \kappa \int_{-\infty}^{+\infty} d Z K(Z)^{1 / 6} \phi_{n}(Z) \phi_{n}(Z)$,

$v_{V}^{2}=\frac{4}{27} \frac{\left(1+N_{f}\right)^{2}}{N_{f}^{2}} v^{2} M_{K K}^{3} R^{3}$.

For the longitudinal modes, the decomposition of $\Phi_{M}$ could be chosen as ${ }^{8}$

$Q_{\mu, m}(x)=\partial_{\mu} Q_{m}(x) U_{K K}, \quad \tilde{\phi}_{m}(Z)=\partial_{Z} \phi_{m}(Z)$,

and the renormalization for $\tilde{\phi}_{m}$ is suggested as

$\kappa v_{V}^{2} U_{K K}^{2} \int_{-\infty}^{+\infty} d Z K(Z)^{1 / 6} \phi_{m}(Z) \phi_{n}(Z)=\delta_{m n}$

then we can obtain the kinetic term of the massive (pseudo) scalar HL-fields from (3.12) (3.16) which is,

$S_{\mathrm{HL}}=-\operatorname{Tr} \int d^{4} x \sum_{n}\left[\partial_{\mu} Q_{n}^{\dagger} \partial^{\mu} Q_{n}+M_{S, n}^{2} Q_{n}^{\dagger} Q_{n}\right]$,

where,

$$
\begin{aligned}
M_{S, n}^{2} & =v_{S}^{2} \kappa \int_{-\infty}^{+\infty} d Z K(Z)^{3 / 2} \phi_{n}(Z) \phi_{n}(Z), \\
v_{S}^{2} & =\frac{64}{2187} \frac{\left(1+N_{f}\right)^{2}}{N_{f}^{2}} v^{2} M_{K K}^{9} R^{9} .
\end{aligned}
$$

Note that $M_{V}, M_{S}$ relate to the mass term of the heavy-light meson fields while $M_{V}, M_{S}$ might be theoretically divergent because they are defined by an integral (3.25). The potential infinities in $M_{V}$ and $M_{S}$ originate from the infinite volume

\footnotetext{
${ }_{8}$ We assume that $\Phi$ is expanded with a pre-factor $U_{K K}$ so that the base functions are all dimensionless.
}

of the spacetime which could always and systematically be removed by the holographic renormalization e.g. in $[17,21$, 40,41]. We give a brief discussion about this in the Appendix D.

\section{The interactions of glueball and heavy-light flavoured mesons}

Since the gravitational perturbation signals the glueball states in holography, let us include the gravitational fluctuations in this section in order to obtain the effective Lagrangian of glueball and mesons. The glueball field could be involved by replacing the metric by $G \rightarrow G+\delta G$ as we discussed in Sect. 1, the interaction of glueball and various mesons will therefore arise once we add the gravitational fluctuations to the terms depended on the metric in the action. Accordingly it only concerns the Yang-Mills action (3.10) and the mass term (3.16) since the Chern-Simons action in (3.3) is independent on the metric. Thus let us first rewrite the Yang-Mills action (3.10) as,

$$
\begin{aligned}
S_{\mathrm{DBI}}^{\mathrm{YM}} & \equiv \sum_{i=1}^{3} S_{i} \equiv S_{\mathrm{L}}+S_{\mathrm{HL}} \\
& +\sum_{i=1}^{3}\left(S_{i}^{H L-L}+S_{i}^{L-G}+S_{i}^{H L-G}+S_{i}^{H L-L-G}\right)
\end{aligned}
$$

where $S_{\mathrm{L}}, S_{\mathrm{HL}}$ refers to the kinetic terms of light and HLmesons given respectively in (3.8) (3.20) and

$$
\begin{aligned}
S_{1}= & -\frac{1}{4}\left(2 \pi \alpha^{\prime}\right)^{2} T_{8} \omega_{4} \operatorname{Tr} \int d^{4} x d Z e^{-\Phi} \sqrt{-g_{(5)}} g^{\mu \rho} g^{\nu \sigma} \\
& \times\left[\left(F_{\mu \nu}-a_{\mu \nu}\right)\left(F_{\rho \sigma}-a_{\rho \sigma}\right)-2 f_{\mu \nu}^{\dagger} f_{\rho \sigma}+b_{\mu \nu} b_{\rho \sigma}\right], \\
S_{2}= & -\frac{1}{2}\left(2 \pi \alpha^{\prime}\right)^{2} T_{8} \omega_{4} \operatorname{Tr} \int d^{4} x d Z e^{-\Phi} \sqrt{-g_{(5)}} \\
& \times\left(g^{\mu \nu} g^{Z Z}-g^{\mu Z} g^{\nu Z}\right)\left[\left(F_{\mu Z}-a_{\mu Z}\right)\left(F_{\nu Z}-a_{\nu Z}\right)\right. \\
& \left.-2 f_{\mu Z}^{\dagger} f_{\nu Z}+b_{\mu Z} b_{\nu Z}\right], \\
S_{3}= & -\left(2 \pi \alpha^{\prime}\right)^{2} T_{8} \omega_{4} \operatorname{Tr} \int d^{4} x d Z e^{-\Phi} \sqrt{-g_{(5)}} g^{\mu \rho} g^{\nu Z} \\
& \times\left[\left(F_{\mu \nu}-a_{\mu \nu}\right)\left(F_{\rho Z}-a_{\rho Z}\right)-2 f_{\mu \nu}^{\dagger} f_{\rho Z}+b_{\mu \nu} b_{\rho Z}\right] .
\end{aligned}
$$

The coupling terms of light mesons and glueball, HL-mesons and glueball, HL- and light meson, light and HL-meson with glueball have been respectively denoted by using the index "L-G" "HL-G" "HL-L" "HL-L-G" in the action (4.1). Note that only the linear terms of the glueball field are valid since 
the gravitational fluctuations are perturbations in the supergravity description. Then the $10 \mathrm{~d}$ metric involving the $11 \mathrm{~d}$ fluctuations is given as,

$$
\begin{aligned}
g_{\mu \nu} & =\frac{r^{3}}{L^{3}}\left[\left(1+\frac{L^{2}}{2 r^{2}} \delta G_{11,11}\right) \eta_{\mu \nu}+\frac{L^{2}}{r^{2}} \delta G_{\mu \nu}\right], \\
g_{44} & =\frac{r^{3} f}{L^{3}}\left[1+\frac{L^{2}}{2 r^{2}} \delta G_{11,11}+\frac{L^{2}}{r^{2} f} \delta G_{44}\right], \\
g_{r r} & =\frac{L}{r f}\left(1+\frac{L^{2}}{2 r^{2}} \delta G_{11,11}+\frac{r^{2} f}{L^{2}} \delta G_{r r}\right), \\
g_{r \mu} & =\frac{r}{L} \delta G_{r \mu}, \quad g_{\Omega \Omega}=\frac{r}{L}\left(\frac{L}{2}\right)^{2}\left(1+\frac{L^{2}}{2 r^{2}} \delta G_{11,11}\right),
\end{aligned}
$$

with the dilaton,

$$
e^{4 \Phi / 3}=\frac{r^{2}}{L^{2}}\left(1+\frac{L^{2}}{r^{2}} \delta G_{11,11}\right) .
$$

We would explicitly derive the general form of the effective action for glueball and mesons by considering the various gravitational fluctuations $\delta G$ in the following subsections. Note that the coupling term for the transverse modes of the HL meson (vector) could be obtained by replacing $\tilde{\phi}_{m}(Z) \rightarrow 0$ and the coupling terms for the longitudinal modes (scalar) can be obtained by replacing $Q_{\mu, m}(x) \rightarrow$ $\partial_{\mu} Q_{m}(x) U_{K K}, \tilde{\phi}_{m}(Z) \rightarrow \partial_{Z} \phi_{m}(Z)$ which could simplify the formulas in the pertinent situations.

\subsection{Involving the exotic scalar glueball}

Let us start with the $10 \mathrm{~d}$ metric (2.7) involving the exotic polarizations of the bulk graviton given in (2.10). Substituting (4.3), (4.4) with (2.10), (3.18) into action (4.2), we can obtain the coupling terms of the glueball and mesons. Although the calculation is very straightforward, the result might be a little messy. Let us keep the quadratic terms of $\Phi_{M}$ as the effective action (4.2), so the interaction terms of glueball and meson are collected as follows, ${ }^{9}$

$$
\begin{gathered}
S_{1}^{H L-L}=\operatorname{Tr} \sum_{m, n} \int d^{4} x d Z \mathcal{A}_{1} \eta^{\mu \rho} \eta^{\nu \sigma} \mathcal{I}_{\mu \nu \rho \sigma}, \\
S_{1}^{L-G_{E}}=\operatorname{Tr} \int d^{4} x d Z\left(\frac{\mathcal{B}_{1}^{E}}{M_{E}^{2}} \partial^{2} G_{E} F_{\mu \nu} F^{\mu \nu}\right. \\
\left.+\frac{\mathcal{C}_{1}^{E}}{M_{E}^{2}} \partial^{\mu} \partial^{\rho} G_{E} F_{\mu \nu} F_{\rho}{ }^{\nu}+\mathcal{D}_{1}^{E} G_{E} F_{\mu \nu} F^{\mu \nu}\right),
\end{gathered}
$$

\footnotetext{
9 We will use $G_{E, D}, T$ to denote the interaction involving the exotic, dilatonic scalar glueball and tensor glueball respectively. The action for the interaction of glueball and $\pi, \rho$ meson in this model is collected in Appendix C.
}

$$
\begin{aligned}
& S_{1}^{H L-G_{E}}=2 \operatorname{Tr} \sum_{m, n} \int d^{4} x d Z \phi_{m} \phi_{n} \\
& \quad \times\left(\frac{\mathcal{B}_{1}^{E}}{M_{E}^{2}} \partial^{2} G_{E} \partial_{[\mu} Q_{\nu], m}^{\dagger} \partial^{[\mu} Q_{, n}^{\nu]}\right. \\
& \quad+\frac{\mathcal{C}_{1}^{E}}{M_{E}^{2}} \eta^{\nu \sigma} \partial^{\mu} \partial^{\rho} G_{E} \partial_{[\mu} Q_{v], m}^{\dagger} \partial_{[\rho} Q_{\sigma], n} \\
& \left.\quad+\mathcal{D}_{1}^{E} G_{E} \partial_{[\mu} Q_{v], m}^{\dagger} \partial^{[\mu} Q_{, n}^{\nu]}\right), \\
& S_{1}^{H L-L-G_{E}}=\operatorname{Tr} \sum_{m, n} \int d^{4} x d Z\left(\frac{\mathcal{B}_{1}^{E}}{M_{E}^{2}} \eta^{\mu \rho} \eta^{\nu \sigma} \partial^{2} G_{E} \mathcal{I}_{\mu \nu \rho \sigma}\right. \\
& \left.\quad+\frac{\mathcal{C}_{1}^{E}}{M_{E}^{2}} \eta^{\nu \sigma} \partial^{\mu} \partial^{\rho} G_{E} \mathcal{I}_{\mu \nu \rho \sigma}+\mathcal{D}_{1}^{E} \eta^{\mu \rho} \eta^{\nu \sigma} G_{E} \mathcal{I}_{\mu \nu \rho \sigma}\right)
\end{aligned}
$$

where

$$
\begin{aligned}
\mathcal{I}_{\mu \nu \rho \sigma}= & \sum_{m, n}\left(-Q_{[\mu, m} Q_{\nu], n}^{\dagger} F_{\rho \sigma}-F_{\mu \nu} Q_{[\rho, m} Q_{\sigma], n}^{\dagger}\right. \\
& +2 \partial_{[\mu} Q_{\nu], m}^{\dagger} A_{[\rho} Q_{\sigma], n}+2 Q_{[\mu, m}^{\dagger} A_{\nu]} \partial_{[\rho} Q_{\sigma], n} \\
& \left.+Q_{[\mu, m}^{\dagger} A_{\nu]} A_{[\rho} Q_{\sigma], n}\right) \phi_{m} \phi_{n} .
\end{aligned}
$$

Note that $\mathcal{I}_{\mu \nu \rho \sigma}$ satisfies the relation $\mathcal{I}_{\mu \nu \rho \sigma}=-\mathcal{I}_{\mu \nu \sigma \rho}=$ $-\mathcal{I}_{\nu \mu \rho \sigma}, \operatorname{Tr} \mathcal{I}_{\mu \nu \rho \sigma}=\operatorname{Tr} \mathcal{I}_{\rho \sigma \mu \nu}$. And we also have,

$$
\begin{aligned}
& S_{2}^{H L-L}=2 M_{K K}^{2} \operatorname{Tr} \sum_{m, n} \int d^{4} x d Z \mathcal{A}_{2} \eta^{\mu \nu} \mathcal{I}_{\mu \nu}, \\
& S_{2}^{L-G_{E}}=M_{K K}^{2} \operatorname{Tr} \int d^{4} x d Z\left[\frac{\mathcal{B}_{2}^{E}}{M_{E}^{2}} \partial^{\mu} \partial^{\nu} G_{E} F_{\mu Z} F_{\nu Z}\right. \\
& \left.+\left(\frac{\mathcal{C}_{2}^{E}}{M_{E}^{2}} \partial^{2} G_{E}+\mathcal{D}_{2}^{E} G_{E}\right) F_{\mu Z} F_{Z}^{\mu}\right], \\
& S_{2}^{H L-G_{E}}=2 M_{K K}^{2} \operatorname{Tr} \sum \int d_{m, n} \int d^{4} x d Z \\
& \quad \times\left[\left(\frac{\mathcal{C}_{2}^{E}}{M_{E}^{2}} \partial^{2} G_{E} \eta^{\mu \nu}+\frac{\mathcal{B}_{2}^{E}}{M_{E}^{2}} \partial^{\mu} \partial^{\nu} G_{E}+\mathcal{D}_{2}^{E} G_{E} \eta^{\mu \nu}\right)\right. \\
& \left.\quad \times\left(U_{K K}^{2} \partial_{\mu} Q_{m}^{\dagger} \partial_{\nu} Q_{n} \tilde{\phi}_{m} \tilde{\phi}_{n}+Q_{\mu, m}^{\dagger} Q_{\nu, m} \phi_{m}^{\prime} \phi_{n}^{\prime}\right)\right],
\end{aligned}
$$

$$
\begin{gathered}
S_{2}^{H L-L-G_{E}}=M_{K K}^{2} \operatorname{Tr} \sum_{m, n} \int d^{4} x d Z\left[\frac{\mathcal{B}_{2}^{E}}{M_{E}^{2}} \partial^{\mu} \partial^{\nu} G_{E} \mathcal{I I}_{\mu \nu}\right. \\
\left.+\frac{\mathcal{C}_{2}^{E}}{M_{E}^{2}} \eta^{\mu \nu} \partial^{2} G_{E} \mathcal{I I}_{\mu \nu}+\mathcal{D}_{2}^{E} G_{E} \eta^{\mu \nu} \mathcal{I I}_{\mu \nu}\right]
\end{gathered}
$$


where

$$
\begin{aligned}
\mathcal{I I}_{\mu \nu}= & \sum_{m, n}\left[-U_{K K} \phi_{m} \tilde{\phi}_{n} Q_{\mu, m} Q_{n}^{\dagger} F_{\nu Z}\right. \\
& +U_{K K} \phi_{m} \tilde{\phi}_{n} Q_{n} Q_{\mu, m}^{\dagger} F_{\nu Z} \\
& -U_{K K} \phi_{m} \tilde{\phi}_{n} F_{\mu Z} Q_{v, m} Q_{n}^{\dagger} \\
& +U_{K K} \phi_{m} \tilde{\phi}_{n} F_{\mu Z} Q_{n} Q_{v, m}^{\dagger} \\
& +2\left(U_{K K} Q_{\mu, m}^{\dagger} A_{Z} \partial_{\nu} Q_{n} \phi_{m} \tilde{\phi}_{n}\right. \\
& -U_{K K}^{2} Q_{m}^{\dagger} A_{\mu} \partial_{\nu} Q_{n} \tilde{\phi}_{m} \tilde{\phi}_{n} \\
& -Q_{\mu, m}^{\dagger} A_{Z} Q_{v, n} \phi_{m} \phi_{n}^{\prime}+U_{K K} Q_{m}^{\dagger} A_{\mu} Q_{v, n} \tilde{\phi}_{m} \phi_{n}^{\prime} \\
& +U_{K K}^{2} \partial_{\mu} Q_{m}^{\dagger} A_{\nu} Q_{n} \tilde{\phi}_{m} \tilde{\phi}_{n} \\
& -U_{K K} Q_{\mu, m}^{\dagger} A_{\nu} Q_{n} \phi_{m}^{\prime} \tilde{\phi}_{n} \\
& -U_{K K} \partial_{\mu} Q_{m}^{\dagger} A_{Z} Q_{v, n} \tilde{\phi}_{m} \phi_{n}+Q_{\mu, m}^{\dagger} A_{Z} Q_{v, n} \phi_{m}^{\prime} \phi_{n} \\
& +U_{K K} Q_{\mu, m}^{\dagger} A_{Z} A_{\nu} Q_{n} \phi_{m} \tilde{\phi}_{n} \\
& -U_{K K}^{2} Q_{m}^{\dagger} \tilde{\phi}_{m} A_{\mu} A_{\nu} Q_{n} \tilde{\phi}_{n} \\
& -Q_{\mu, m}^{\dagger} A_{Z}^{2} Q_{v, n} \phi_{m} \phi_{n} \\
& \left.\left.+U_{K K} Q_{m}^{\dagger} A_{\mu} A_{Z} Q_{v, n} \tilde{\phi}_{m} \phi_{n}\right)\right] .
\end{aligned}
$$

For the terms in $S_{3}$, respectively they are,

$$
\begin{aligned}
& S_{3}^{L-G_{E}}=\frac{M_{K K}^{2}}{M_{E}^{2}} \operatorname{Tr} \int d^{4} x d Z c(Z) \partial_{\sigma} G_{E} F^{\rho \sigma} F_{\rho Z}, \\
& S_{3}^{H L-G_{E}}=\frac{2 M_{K K}^{2}}{M_{E}^{2}} \operatorname{Tr} \sum_{m, n} \int d^{4} x d Z c(Z) \partial_{\sigma} G_{E} \\
& \quad \times\left(\phi_{m} \tilde{\phi}_{n} U_{K K} \partial^{[\rho} Q_{,{ }_{m}}^{\sigma \dagger \dagger} \partial_{\rho} Q_{n}-\phi_{m} \phi_{n}^{\prime} \partial^{[\rho} Q_{, m}^{\sigma] \dagger} Q_{\rho, n}\right), \\
& S_{3}^{H L-L-G_{E}}=\frac{M_{K K}^{2}}{M_{E}^{2}} \operatorname{Tr} \sum_{m, n} \int d^{4} x d Z c(Z) \partial_{\sigma} G_{E} \\
& \quad \times\left[-\phi_{m} \phi_{n} Q_{, m}^{[\rho} Q_{, n}^{\sigma] \dagger} F_{\rho Z}-F^{\rho \sigma} Q_{\rho, m} Q_{n}^{\dagger} U_{K K} \phi_{m} \tilde{\phi}_{n}\right. \\
& \quad+F^{\rho \sigma} Q_{n} Q_{\rho, m}^{\dagger} U_{K K} \phi_{m} \tilde{\phi}_{n}+2\left(U_{K K} \partial^{[\rho} Q_{, m}^{\sigma \dagger \dagger} \partial_{\rho} Q_{n} \phi_{m} \tilde{\phi}_{n}\right. \\
& \quad-\partial^{[\rho} Q_{, m}^{\sigma] \dagger} Q_{\rho, n} \phi_{m} \phi_{n}^{\prime} \\
& \quad+U_{K K} Q_{, m}^{[\rho \dagger} A^{\sigma]} \partial_{\rho} Q_{n} \phi_{m} \tilde{\phi}_{n}-Q_{, m}^{[\rho \dagger} A^{\sigma]} Q_{\rho, n} \phi_{m} \phi_{n}^{\prime} \\
& \quad+U_{K K} \partial^{[\rho} Q_{, m}^{\sigma] \dagger} A_{\rho} Q_{n} \phi_{m} \tilde{\phi}_{n} \\
& \quad-\partial^{[\rho} Q_{, m}^{\sigma] \dagger} A_{Z} Q_{\rho, n} \phi_{m} \phi_{n}+U_{K K} Q_{, m}^{[\rho \dagger} A^{\sigma]} A_{\rho} Q_{n} \phi_{m} \tilde{\phi}_{n} \\
& \left.\left.\quad-Q_{, m}^{[\rho \dagger} A^{\sigma]} A_{Z} Q_{\rho, n} \phi_{m} \phi_{n}\right)\right],
\end{aligned}
$$

and all the coefficients are given as,

$$
\mathcal{A}_{1}=-\frac{\kappa}{2} K^{-1 / 3}, \quad \mathcal{B}_{1}^{E}=\frac{1}{4} \kappa \tilde{H}_{E} K^{-1 / 3},
$$

$$
\begin{aligned}
\mathcal{C}_{1}^{E} & =-\kappa \tilde{H}_{E} K^{-1 / 3}, \quad \mathcal{D}_{1}^{E}=-\frac{1}{16} \frac{5 K-14}{5 K-2} \kappa H_{E} K^{-1 / 3}, \\
\mathcal{A}_{2} & =-\kappa K, \mathcal{B}_{2}^{E}=-\kappa K \tilde{H}_{E}, \quad \mathcal{C}_{2}^{E}=\frac{1}{2} \kappa K \tilde{H}_{E}, \\
\mathcal{D}_{2}^{E} & =-\frac{3}{8} \frac{5 K+2}{5 K-2} \kappa K H_{E}, \\
c(Z) & =60 \kappa \frac{Z K}{(5 K-2)^{2}} H_{E}, \quad \bar{H}_{E}=\left(\frac{1}{4}+\frac{3}{5 K-2}\right) H_{E} .
\end{aligned}
$$

Additionally we have another coupling from the mass terms (3.16) which is,

$$
\begin{aligned}
& S_{m}^{H L-G_{E}}=-\frac{v_{V}^{2}}{M_{E}^{2}} \operatorname{Tr} \sum_{m \cdot n} \int d^{4} x \\
& \quad \times\left[s_{m n}^{(1)} \partial^{2} G_{E} Q_{\mu, m}^{\dagger} Q_{, n}^{\mu}+s_{m n}^{(2)} \partial^{\mu} \partial^{v} G_{E} Q_{\mu, m}^{\dagger} Q_{v, n}\right. \\
& \left.\quad+s_{m n}^{(3)} M_{E}^{2} G_{E} Q_{\mu, m}^{\dagger} Q_{, n}^{\mu}\right] \\
& \quad+\frac{v_{S}^{2}}{M_{E}^{2}} \operatorname{Tr} \sum_{m \cdot n} \int d^{4} x\left[s_{m n}^{(4)} \partial^{2} G_{E} Q_{m}^{\dagger} Q_{n}+s_{m n}^{(5)} M_{E}^{2} G_{E} Q_{m}^{\dagger} Q_{n}\right] \\
& \quad+\alpha_{E}^{2} \operatorname{Tr} \sum_{m . n} \int d^{4} x s_{m n}^{(6)} M_{K K} \partial^{\mu} G_{E}\left(Q_{\mu, m}^{\dagger} Q_{n}+Q_{n}^{\dagger} Q_{\mu, m}\right),
\end{aligned}
$$

where $\alpha_{E}=\frac{\left(1+N_{f}\right) v}{N_{f} M_{E}} \frac{v_{S}}{v_{V}}$ and the coefficients are,

$s_{m n}^{(1)}=\frac{\kappa}{2} \int d Z K^{1 / 6} \tilde{H}_{E} \phi_{m} \phi_{n}$,

$s_{m n}^{(2)}=-\kappa \int d Z K^{1 / 6} \tilde{H}_{E} \phi_{m} \phi_{n}$,

$s_{m n}^{(3)}=-\kappa \int d Z \frac{5 Z^{2} H_{E}}{2(5 K-2)} K^{1 / 6} \phi_{m} \phi_{n}$,

$s_{m n}^{(4)}=-\frac{\kappa}{2} \int d Z K^{3 / 2} \tilde{H}_{E} \tilde{\phi}_{m} \tilde{\phi}_{n}$

$s_{m n}^{(5)}=\kappa \int d Z \frac{15 K^{5 / 2} H_{E}}{4(5 k-2)} \tilde{\phi}_{m} \tilde{\phi}_{n}$,

$s_{m n}^{(6)}=-\kappa \int d Z \frac{10 Z K^{3 / 2}}{(5 K-2)^{2}} H_{E} \phi_{m} \tilde{\phi}_{n}$.

The total action of the exotic scalar glueball and various mesons could be obtained by collecting all the terms in (4.1) and (4.19) with the kinetic term of the glueball in (2.12).

4.2 Involving the dilatonic scalar glueball

The effective action of the dilatonic glueball and mesons could be directly obtained by plugging (4.3), (4.4) with the dilatonic polarizations (2.14) into action (4.2). We find that the interaction terms $S_{i}^{H L-L}$ in (4.2) remains since they are independent on the metric fluctuations. So the interaction terms of dilatonic glueball and mesons can be collected as 
$S_{\text {int }}^{G_{D}}=\sum_{i=1}^{2}\left(S_{i}^{L-G_{D}}+S_{i}^{H L-G_{D}}+S_{i}^{H L-L-G_{D}}\right)+S_{m}^{H L-G_{D}}$,

where $S_{i}^{L-G_{D}}, S_{i}^{H L-G_{D}}, S_{i}^{H L-L-G_{D}}$ takes the same formulas as in the case of the exotic scalar glueball while the coefficients have to be replaced by $\mathcal{B}_{1,2}^{E}, \mathcal{C}_{1,2}^{E}, \mathcal{D}_{1,2}^{E} \rightarrow$ $\mathcal{B}_{1,2}^{D}, \mathcal{C}_{1,2}^{D}, \mathcal{D}_{1,2}^{D}$. And the corresponding coefficients are given as,

$\mathcal{B}_{1}^{D}=\frac{1}{4} \kappa H_{D} K^{-1 / 3}, \quad \mathcal{C}_{1}^{D}=-\kappa H_{D} K^{-1 / 3}$,

$\mathcal{D}_{1}^{D}=\frac{3}{4} \kappa H_{D} K^{-1 / 3} \quad \mathcal{B}_{2}^{D}=-\kappa K H_{D}$,

$\mathcal{C}_{2}^{D}=\frac{1}{2} \kappa K H_{D}, \quad \mathcal{D}_{2}^{D}=\mathcal{C}_{2}^{D}$.

The coupling term $S_{m}^{H L-G_{D}}$ for the dilatonic glueball from the mass terms (3.16) also takes the same formula as in (4.19) while the coefficients need to be replaced as $s_{m n}^{(1,2 \ldots 6)} \rightarrow$

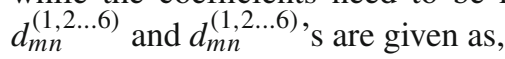

$d_{m n}^{(1)}=\frac{\kappa}{2} \int d Z K^{1 / 6} H_{D} \phi_{m} \phi_{n}$,

$d_{m n}^{(2)}=-\kappa \int d Z K^{1 / 6} H_{D} \phi_{m} \phi_{n}$,

$d_{m n}^{(3)}=2 \kappa \int d Z K^{1 / 6} H_{D} \phi_{m} \phi_{n}$,

$d_{m n}^{(4)}=\frac{\kappa}{2} \int d Z K^{3 / 2} H_{D} \tilde{\phi}_{m} \tilde{\phi}_{n}$

$d_{m n}^{(5)}=\kappa \int d Z K^{3 / 2} H_{D} \tilde{\phi}_{m} \tilde{\phi}_{n}, d_{m n}^{(6)}=0$.

So the total action for the interaction of dilatonic scalar glueball and meson could also be collected from (4.5)-(4.17) and (4.19) while the coefficients are replaced by (4.22) and (4.23).

\subsection{Involving the tensor glueball}

The interaction terms of tensor glueball and mesons can be collected by inserting (4.3), (4.4) with the polarizations (2.15) into (3.11) and (3.16). As before, let us rewrite the interaction terms involving the tensor glueball in the action as,

$S_{\text {int }}^{T}=\sum_{i=1}^{2}\left(S_{i}^{L-T}+S_{i}^{H-T}+S_{i}^{H L-L-T}\right)+S_{m}^{H L-T}$,

where their explicit formulas are collected as,

$S_{1}^{L-T}=\operatorname{Tr} \int d^{4} x d Z B_{1}^{T} T^{\mu \rho} \eta^{\nu \sigma} F_{\mu \nu} F_{\rho \sigma}$,

$$
\begin{gathered}
S_{1}^{H L-T}=2 \operatorname{Tr} \sum_{m, n} \int d^{4} x d Z B_{1}^{T} \phi_{m} \phi_{n} T^{\nu \sigma} \eta^{\mu \rho} \\
\partial_{[\mu} Q_{v, m]}^{\dagger} \partial_{[\rho} Q_{\sigma, n]}, \\
S_{1}^{H L-L-T}=\operatorname{Tr} \sum_{m, n} \int d^{4} x d Z B_{1}^{T} T^{\nu \sigma} \eta^{\mu \rho} \mathcal{I}_{\mu \nu \rho \sigma},
\end{gathered}
$$

and

$$
\begin{aligned}
S_{2}^{L-T}= & M_{K K}^{2} \operatorname{Tr} \int d^{4} x d Z \mathcal{B}_{2}^{T} T^{\mu \nu} F_{\mu Z} F_{\nu Z} \\
S_{2}^{H L-T}= & 2 M_{K K}^{2} \operatorname{Tr} \sum_{m, n} \int d^{4} x d Z \mathcal{B}_{2}^{T} T^{\mu \nu} \\
& \times\left(U_{K K}^{2} \partial_{\mu} Q_{m}^{\dagger} \partial_{\nu} Q_{n} \tilde{\phi}_{m} \tilde{\phi}_{n}+Q_{\mu, m}^{\dagger} Q_{v, m} \phi_{m}^{\prime} \phi_{n}^{\prime}\right),
\end{aligned}
$$

$S_{2}^{H L-L-T}=M_{K K}^{2} \operatorname{Tr} \int d^{4} x d Z \mathcal{B}_{2}^{T} T^{\mu \nu} \mathcal{I I}_{\mu \nu}$.

The coupling from the mass term (3.16) is given as,

$S_{m}^{H L-T}=-\sum_{m . n} v_{V}^{2} t_{m n} \operatorname{Tr} \int d^{4} x T^{\mu v} Q_{\mu, m}^{\dagger} Q_{v, n}$,

where the corresponding coefficients in above actions are,

$$
\begin{aligned}
\mathcal{B}_{1}^{T} & =-\kappa K^{-1 / 3} H_{T}, \mathcal{B}_{2}^{T}=-\kappa K H_{T}, t_{m n} \\
& =-\kappa \int d Z K^{1 / 6} H_{D} \phi_{m} \phi_{n} .
\end{aligned}
$$

\section{Interaction of glueball and the lowest heavy-light meson in holography}

Since the lowest heavy-light meson is recognized as Dmeson, let us identify the lowest heavy-light meson field in our theory as the lowest D-meson field (i.e. the $D^{0}$ meson) and investigate the decay/production of glueball to/from them holographically in this section. And it may provide a holographic way to study the $D^{0}-\bar{D}^{0}$ or $B_{s}-\bar{B}_{s}$ oscillation/annihilation. Additionally we also evaluate the decay rate of glueball to light mesons $\rho$ and $\pi$ in Appendix $\mathrm{C}$ as a comparison by using the parameters given in Sect. 5.1.

5.1 The mode eigenfunctions and the choice of parameters

Let outline the parameters and formulas to investigate the decay/production of glueball in this section. For the vector HL-meson fields, the eigenfunctions $\left\{\phi_{m}\right\}$ (3.21) have to satisfy the normalization condition (3.21) which is exactly the same as the normalization of $\left\{\psi_{n}\right\}$ for $n \geq 1$ presented in [12], 
Table 2 Glueball mass spectrum $m_{n}^{2}$ of $\mathrm{AdS}_{7}$ in the units of $r_{K K}^{2} / L^{4}=M_{K K}^{2} / 9$ from [26]

\begin{tabular}{|c|c|c|c|c|c|c|}
\hline $\begin{array}{l}\text { Mode } \\
J^{P C}\end{array}$ & $\begin{array}{l}\mathrm{S}_{4} \\
0^{+}+\end{array}$ & $\begin{array}{l}\mathrm{T}_{4} \\
0^{+}+/ 2^{+}+\end{array}$ & $\begin{array}{l}\mathrm{V}_{4} \\
0^{-}+\end{array}$ & $\begin{array}{l}\mathrm{N}_{4} \\
1^{+}-\end{array}$ & $\begin{array}{l}\mathrm{M}_{4} \\
1^{-}-\end{array}$ & $\begin{array}{l}\mathrm{L}_{4} \\
0^{+}+\end{array}$ \\
\hline$n=0$ & 7.30835 & 22.0966 & 31.9853 & 53.3758 & 83.0449 & 115.002 \\
\hline$n=1$ & 46.9855 & 55.5833 & 72.4793 & 109.446 & 143.581 & 189.632 \\
\hline$n=2$ & 94.4816 & 102.452 & 126.144 & 177.231 & 217.397 & 227.283 \\
\hline$n=3$ & 154.963 & 162.699 & 193.133 & 257.959 & 304.531 & 378.099 \\
\hline$n=4$ & 228.709 & 236.328 & 273.482 & 351.895 & 405.011 & 492.171 \\
\hline
\end{tabular}

$$
\begin{aligned}
2 \kappa \int_{-\infty}^{+\infty} d Z K(Z)^{-1 / 3} \psi_{m}(Z) \psi_{n}(Z) & =\delta_{m n}, \\
2 \kappa \int_{-\infty}^{+\infty} d Z K(Z) \partial_{Z} \psi_{m} \partial_{Z} \psi_{n} & =\lambda_{n} \delta_{m n} .
\end{aligned}
$$

From these, the functions $\left\{\psi_{n}\right\}$ for $n \geq 1$ could be generated by the following eigen equation,

$-K^{1 / 3} \partial_{Z}\left(K \partial_{Z} \psi_{n}\right)=\lambda_{n} \psi_{n}, \psi_{n}(Z \rightarrow \pm \infty)=0$,

Thus it is seen that we can choose $\phi_{n}=\frac{\psi_{n}}{\sqrt{2}}$ for all $n \geq 1$. For the scalar HL-meson field, we can choose $\phi_{n}=\frac{1}{\sqrt{2}} v_{V}^{-1} U_{K K}^{-1} K(Z)^{-1 / 4} \psi_{n}$ according to (3.23). Note that there still exists a function $\tilde{\phi}_{0}$ orthogonal to $\left\{\tilde{\phi}_{n}\right\}$ for all $n \geq 1$ which is approximately given as,

$\tilde{\phi}_{0}=\frac{1}{\sqrt{2}} \psi_{0}^{\prime}(Z)=\frac{1}{2 \sqrt{\pi \kappa}} \frac{1}{U_{K K} v_{V}} \frac{1}{K(Z)}$

while $\psi_{0}$ does not satisfy the eigen equation (5.2). So the complete set of eigenfunctions $\left\{\phi_{m}\right\}$ can be obtained by redefining $\phi_{m}=\frac{\psi_{m+1}}{\sqrt{2}}$ for all $m \geq 0$ thus $\Lambda_{0}=1, \Lambda_{m}=$ $\lambda_{m}, m>0$. Hence we are going to continue the discussion with this definition.

In order to match the experimental value of the $\rho$ meson mass $m_{\rho}=\sqrt{\lambda_{1}} M_{K K} \simeq 776 \mathrm{MeV}$ where $\lambda_{1}$ is numerically evaluated as $\lambda_{1}=0.669314 \ldots$, we first need to fix the Kaluza-Klein mass to $M_{K K}=949 \mathrm{MeV}$. Then let us identify the lowest mode of the heavy-light meson field $Q_{\mu, n=0}, Q_{n=0}$ as the vector meson $D_{\mu}^{0}$ and the pseudoscalar meson $D^{0}$ whose mass is given as $M\left(D_{\mu}^{0}\right) \simeq$ $2007 \mathrm{MeV}, M\left(D^{0}\right) \simeq 1865 \mathrm{MeV}$ by experiments. In this sense, we further consider the case of $N_{f}=2$ for D-meson, so the parameters in (3.20), (3.25) can be numerically fixed as

$$
\begin{aligned}
M_{S} & \simeq 1754 \mathrm{MeV}, M_{V} \simeq 2007 \mathrm{MeV}, M_{K K} R \simeq 1.048, \\
v & \simeq 35195.4 \lambda^{-1} N_{c}^{-1} M_{K K} .
\end{aligned}
$$

On the other hand, the glueball mass in holography is determined by solving the eigen equations (2.11), (2.16) for exotic, dilatonic and tensor glueball respectively. For the reader's convenience, we collect the mass spectrum of various glueballs in Table 2. It is clear that the glueball decay occurs when the mass relation $m_{\text {glueball }} / m_{\mathrm{D}-\text { meson }} \simeq 2$ is satisfied because the effective holographic action we discussed is quadratic of the heavy-light meson fields. Accordingly we will choose the excited mass of $n=3$ as $M_{E}=\sqrt{154.963 / 9} M_{K K} \simeq$ $3938 \mathrm{MeV}, M_{D}=M_{T}=\sqrt{162.699 / 9} M_{K K} \simeq 4035 \mathrm{MeV}$ for the exotic, dilatonic and tensor glueball respectively. With these values for the parameters, the boundary condition for the eigen equations (2.11), (2.16) is numerically evaluated as,

$$
\begin{aligned}
H_{E}\left(r_{K K}\right)^{-1} & \simeq 0.00692402 \lambda^{1 / 2} N_{c} M_{K K}, \\
H_{D, T}\left(r_{K K}\right)^{-1} & \simeq 0.0211777 \lambda^{1 / 2} N_{c} M_{K K} .
\end{aligned}
$$

Beside, the formula of the decay width is given as,

$\Gamma=\frac{1}{2 M_{G}} \int d \Pi_{n} \mid\left.\mathcal{M}\{G \rightarrow$ hadrons $\}\right|^{2}$,

where $\mathcal{M}$ refers to the associated amplitude and

$d \Pi_{n}=\left(\prod_{f} \frac{d^{3} \mathbf{p}_{f}}{(2 \pi)^{3}} \frac{1}{2 E_{f}}\right)(2 \pi)^{4} \delta^{4}\left(P_{G}-\sum_{f} p_{f}\right)$,

is the volume element of the n-body phase space. And we will use the parameters above with (5.6) to study the decay of one glueball into the lowest D-mesons.

\subsection{Glueball decay into the lowest scalar $D^{0} \bar{D}^{0}$ meson}

In this section, let us compute the decay/production width of the glueball to two lowest heavy-light mesons i.e. the (pseudo) scalar mesons $\left(D^{0} \bar{D}^{0}\right)$. The expression of the width (5.6) for the scalar glueball into $D^{0} \bar{D}^{0}$ could be simplified as, ${ }^{10}$

$\Gamma_{G_{E, D} \rightarrow D \bar{D}}=\frac{3|\mathbf{p}|}{16 \pi M_{E, D}^{2}}\left|\mathcal{M}_{E, D}\right|^{2}$,

\footnotetext{
${ }^{10}$ We have employed the same convention as those in [26].
} 
where $|\mathbf{p}|=\sqrt{\left(\frac{M_{E, D}}{2}\right)^{2}-M_{D^{0}}^{2}}$ represents the 3-momentum of one $D^{0}$ in the rest frame of the glueball. For the tensor glueball we however need the average over its polarizations. Hence let us integrate over the orientation of the coordinates by choosing a fixed polarization $\epsilon^{11}=-\epsilon^{22}=1$. So it leads to the decay width as.

$$
\begin{aligned}
\Gamma_{G_{T} \rightarrow D \bar{D}} & =\frac{3|\mathbf{p}|}{16 \pi M_{T}^{2}} \int \frac{d \Omega}{4 \pi}\left|\mathcal{M}_{T}\right|^{2}, \\
|\mathbf{p}| & =\sqrt{\left(\frac{M_{T}}{2}\right)^{2}-M_{D^{0}}^{2}} .
\end{aligned}
$$

Note that $\left(D^{0} \bar{D}^{0}\right)$ will be denoted as $(D \bar{D})$ for simplicity in the following discussion.

\subsubsection{Exotic scalar glueball}

The relevant part of the action involving the exotic glueball and $D^{0} \bar{D}^{0}$ can be collected from (4.5) to (4.19) which is,

$$
\begin{aligned}
S^{G_{E} \rightarrow D \bar{D}}= & \operatorname{Tr} \int d^{4} x\left[c_{2}^{E} \partial^{2} G_{E} \partial_{\mu} \bar{D} \partial^{\mu} D\right. \\
& +b_{2}^{E} \partial^{\mu} \partial^{\nu} G_{E} \partial_{\mu} \bar{D} \partial_{\nu} D+d_{2}^{E} G_{E} \partial_{\mu} \bar{D} \partial^{\mu} D \\
& \left.+\frac{v_{S}^{2}}{M_{E}^{2}} s_{00}^{(4)} \partial^{2} G_{E} \bar{D} D+v_{S}^{2} s_{00}^{(5)} G_{E} \bar{D} D\right],
\end{aligned}
$$

where the coupling constants could be numerically evaluated as,

$$
\begin{aligned}
c_{2}^{E} & =\frac{2 M_{K K}^{2} U_{K K}^{2}}{M_{E}^{2}} \int d Z \mathcal{C}_{2}^{E} \tilde{\phi}_{0} \tilde{\phi}_{0} \simeq 0.403 \lambda^{-1 / 2} N_{c}^{-1} M_{K K}^{-3}, \\
b_{2}^{E} & =\frac{2 M_{K K}^{2} U_{K K}^{2}}{M_{E}^{2}} \int d Z \mathcal{B}_{2}^{E} \tilde{\phi}_{0} \tilde{\phi}_{0} \simeq-0.806 \lambda^{-1 / 2} N_{c}^{-1} M_{K K}^{-3}, \\
d_{2}^{E} & =2 M_{K K}^{2} U_{K K}^{2} \int d Z \mathcal{D}_{2}^{E} \tilde{\phi}_{0} \tilde{\phi}_{0} \simeq-9.432 \lambda^{-1 / 2} N_{c}^{-1} M_{K K}^{-1}, \\
s_{00}^{(4)} & \simeq-12.020 \lambda^{-1 / 2} N_{c}^{-1} M_{K K}^{-1}, \quad s_{00}^{(5)} \\
& \simeq-21.749 \lambda^{-1 / 2} N_{c}^{-1} M_{K K}^{-1} .
\end{aligned}
$$

By omitting the terms which vanishes when $G_{E}$ is onshell in (5.10), the amplitude is calculated as,

$$
\begin{aligned}
\left|\mathcal{M}_{E}\right|= & \mid\left[2 b_{2}^{E} M_{E}^{2}+2\left(c_{2}^{E} M_{E}^{2}+d_{2}^{E}\right)\right] p_{0} q_{0} \\
& -2\left(c_{2}^{E} M_{E}^{2}+d_{2}^{E}\right) \mathbf{p} \cdot \mathbf{q}+v_{S}^{2}\left[s_{00}^{(4)}+s_{00}^{(5)}\right] \mid \\
= & \left|\frac{M_{E}^{2}}{4}\left[2 b_{2}^{E} M_{E}^{2}+4\left(c_{2}^{E} M_{E}^{2}+d_{2}^{E}\right)\right]+v_{S}^{2}\left[s_{00}^{(4)}+s_{00}^{(5)}\right]\right|,
\end{aligned}
$$

and the decay/production rate is accordingly obtained as,

$$
\begin{aligned}
\Gamma_{G_{E} \rightarrow D \bar{D}} / M_{E}= & \frac{3|\mathbf{p}|}{16 \pi M_{E}^{3}}\left|\mathcal{M}_{E}\right|^{2} \simeq 14.654 \lambda^{-1} N_{c}^{-2} \\
& +\mathcal{O}\left(N_{c}^{-4}\right) .
\end{aligned}
$$

\subsubsection{Dilatonic scalar glueball}

For the decay of the dilatonic glueball, we have the same formula for the action collected from (4.5) to (4.19),

$$
\begin{aligned}
S^{G_{D} \rightarrow D \bar{D}}= & \operatorname{Tr} \int d^{4} x\left[c_{2}^{D} \partial^{2} G_{D} \partial_{\mu} \bar{D} \partial^{\mu} D\right. \\
& +b_{2}^{D} \partial^{\mu} \partial^{\nu} G_{D} \partial_{\mu} \bar{D} \partial_{\nu} D \\
& +d_{2}^{D} G_{D} \partial_{\mu} \bar{D} \partial^{\mu} D \\
& \left.+\frac{v_{S}^{2}}{M_{E}^{2}} d_{00}^{(4)} \partial^{2} G_{D} \bar{D} D+v_{S}^{2} d_{00}^{(5)} G_{D} \bar{D} D\right]
\end{aligned}
$$

where the coupling constants are numerically evaluated as,

$$
\begin{aligned}
c_{2}^{D} & =\frac{2 M_{K K}^{2} U_{K K}^{2}}{M_{D}^{2}} \int d Z \mathcal{C}_{2}^{D} \tilde{\phi}_{0} \tilde{\phi}_{0} \simeq 0.105 \lambda^{-1 / 2} N_{c}^{-1} M_{K K}^{-3}, \\
b_{2}^{D} & =\frac{2 M_{K K}^{2} U_{K K}^{2}}{M_{D}^{2}} \int d Z \mathcal{B}_{2}^{D} \tilde{\phi}_{0} \tilde{\phi}_{0} \simeq-0.211 \lambda^{-1 / 2} N_{c}^{-1} M_{K K}^{-3}, \\
d_{2}^{D} & =2 M_{K K}^{2} U_{K K}^{2} \int d Z \mathcal{D}_{2}^{D} \tilde{\phi}_{0} \tilde{\phi}_{0} \simeq 1.907 \lambda^{-1 / 2} N_{c}^{-1} M_{K K}^{-1}, \\
d_{00}^{(4)} & \simeq 7.859 \lambda^{-1 / 2} N_{c}^{-1} M_{K K}^{-1}, \quad d_{00}^{(5)} \simeq 6.454 \lambda^{-1 / 2} N_{c}^{-1} M_{K K}^{-1} .
\end{aligned}
$$

Omitting the terms when $G_{D}$ is onshell in (5.10), the amplitude is calculated as,

$$
\begin{aligned}
\left|\mathcal{M}_{D}\right|= & \mid\left[2 b_{2}^{D} M_{D}^{2}+2\left(c_{2}^{D} M_{D}^{2}+d_{2}^{D}\right)\right] p_{0} q_{0} \\
& -2\left(c_{2}^{D} M_{D}^{2}+d_{2}^{D}\right) \mathbf{p} \cdot \mathbf{q}+v_{S}^{2}\left[d_{00}^{(4)}+d_{00}^{(5)}\right] \mid \\
= & \left|\frac{M_{D}^{2}}{4}\left[2 b_{2}^{D} M_{D}^{2}+4\left(c_{2}^{D} M_{D}^{2}+d_{2}^{D}\right)\right]+v_{S}^{2}\left[d_{00}^{(4)}+d_{00}^{(5)}\right]\right|,
\end{aligned}
$$

which leads to the decay/production rate as,

$$
\begin{gathered}
\Gamma_{G_{D} \rightarrow D \bar{D}} / M_{D}=\frac{3|\mathbf{p}|}{8 \pi M_{D}^{3}}\left|\mathcal{M}_{D}\right|^{2} \\
\simeq 0.741 \lambda^{-1} N_{c}^{-2}+\mathcal{O}\left(N_{c}^{-4}\right) .
\end{gathered}
$$




\section{Tensor glueball}

The action involving the tensor glueball and $D \bar{D}$ is very simple,

$$
S^{H L-G_{T}}=b_{2}^{T} \operatorname{Tr} \int d^{4} x T^{\mu \nu} \partial_{\mu} \bar{D} \partial_{\nu} D,
$$

where the coupling constant is,

$$
\begin{aligned}
b_{2}^{T} & =2 M_{K K}^{2} U_{K K}^{2} \int d Z \mathcal{B}_{2}^{T} \tilde{\phi}_{0} \tilde{\phi}_{0} \\
& \simeq-9.343 \lambda^{-1 / 2} N_{c}^{-1} M_{K K}^{-1} .
\end{aligned}
$$

Then we could obtain the amplitude when $T_{\mu \nu}$ is onshell as,

$$
\left|\mathcal{M}_{T}\right|=\left|2 b_{2}^{T}\left(p_{x}^{2}-p_{y}^{2}\right)\right|,
$$

therefore the decay/production rate is evaluated as,

$$
\Gamma_{T \rightarrow D \bar{D}} / M_{T}=\frac{3|\mathbf{p}|}{8 \pi M_{T}^{3}} \int \frac{d \Omega}{4 \pi}\left|\mathcal{M}_{T}\right|^{2} \simeq 0.025 \lambda^{-1} N_{c}^{-2} .
$$

\section{Summary and discussion}

By using the Witten-Sakai-Sugimoto model, we study the interaction of glueball and heavy-light flavoured mesons in holography then extend the calculations of glueball decay/production in $[25,26]$ with heavy flavour. The model contains only one free dimensional parameter $M_{K K}$ since it is a top-down approach of string theory. The glueball fields are identified as the various fluctuations of the bulk geometry produced by the $N_{c}$ compactified D4-branes and the meson fields are created by the open strings living in the $N_{f}$ probe D8/D8-branes. Particularly we construct the D4/D8 configuration as in $[28,29,37,38]$ by introducing an extra pair of probe $\mathrm{D} 8 / \overline{\mathrm{D} 8}$-branes as "heavy flavour" which is separated from the other $N_{f} \mathrm{D} 8 / \overline{\mathrm{D} 8}$-branes with a heavy-light string stretched between them. In this configuration the multiplets produced by the heavy-light string could be identified as the heavy-light flavoured mesons so that the model is able to include their interactions with glueball.

Motivated by the heavy-flavoured hadron-anti hadron oscillation [32,33], we numerically evaluate the three-hadron coupling constants between the various glueballs (the exotic, dilatonic scalar and tensor glueballs) and the lowest heavylight flavoured mesons ( $D^{0}$ meson) then compute the associated decay widths/rates (the production of $D^{0} \bar{D}^{0}$ ) in this model. As an effective theory, the production of $D^{0} \bar{D}^{0}$ would be remarkable to study the $D^{0}-\bar{D}^{0}$ oscillation [32] in holography. And we find the decay/production widths of glueballs are parametrically suppressed by the factor $\lambda^{-1} N_{c}^{-2}$ in the large $N_{c}$ limit while the results vary substantially for the different decay channels. So it could not give the picture of "universal narrowness" although the heavy flavour and large $N_{c}$ limitation are considered in the model. However on the other hand, the decay/production widths of the lightest glueball to the lowest light mesons in this model turn out to be consistent with the experimental data of the $f_{0}(1500)$ decay $[25,26]$, thus our calculation with heavy flavour could be a further prediction of glueball-meson interaction although the experimental evidence are currently insufficient. ${ }^{11}$ Besides, the decay/production rate of the exotic scalar glueball is turned out to have a larger width than that of the heavier dilatonic mode according to our calculation, so our results support partially the conjecture proposed in [26] that is the lowest glueball model begins with the dilatonic mode and the exotic scalar glueball should be discarded.

Noteworthily phenomenological models usually require very large gluon condensates in order to admit only narrow glueball states while the value of the gluon condensate is quite small in the WSS model. Nevertheless it could be interesting to introduce the gluon condensates into a top-down approach of holography as [43-45] so that it would be able to compare with the phenomenological models.

We finally comments that the mixing of glueballs with $q \bar{q}$ meson states would be also interesting to study since the signatures of glueball content can be strongly obscured by the mixing. But it might probably require more difficult stringy corrections which are not captured by the effective action following from the WSS model in holography. Nonetheless this would be more pivotal to identify the glueball in the future study.

Acknowledgements I would like to thank Anton Rebhan, Josef Leutgeb and Kazem Bitaghsir for valuable comment and discussion. This work is supported by the National Natural Science Foundation of China (NSFC) under Grant No. 11947008, the research startup foundation of Dalian Maritime University in 2019 under Grant No. 02502608 and the Fundamental Research Funds for the Central Universities under Grant No. 3132020174, also supported in part by the National Natural Science Foundation of China (NSFC) under Grant No. 11535012.

Data Availability Statement This manuscript has no associated data or the data will not be deposited. [Authors' comment: This is a totally theoretical work, so the calculation and analysis are all based on the current knowledge about physics theory. Thus there is not any data in this work.]

Open Access This article is licensed under a Creative Commons Attribution 4.0 International License, which permits use, sharing, adaptation, distribution and reproduction in any medium or format, as long as you give appropriate credit to the original author(s) and the source, provide a link to the Creative Commons licence, and indicate if changes were made. The images or other third party material in this article

\footnotetext{
11 The $X$ (3020) [42] is the only one glueball candidate whose energy is close to the glueballs' discussed in our manuscript.
} 
are included in the article's Creative Commons licence, unless indicated otherwise in a credit line to the material. If material is not included in the article's Creative Commons licence and your intended use is not permitted by statutory regulation or exceeds the permitted use, you will need to obtain permission directly from the copyright holder. To view a copy of this licence, visit http://creativecomm ons.org/licenses/by/4.0/.

Funded by SCOAP ${ }^{3}$.

\section{Appendix A: The 10d equations of motion in the bulk supergravity}

The WSS model is obtained by the Kaluza-Klein reduction of the $11 \mathrm{~d}$ supergravity on a cycle. Since the supersymmetry is broken down in the model, the bulk geometry of $N_{c}$ D4branes in the large $N_{c}$ limit is determined by the bosonic part of the type IIA supergravity whose action is given as,

$$
\begin{aligned}
S_{\text {IIA }}= & \frac{1}{2 k_{10}^{2}} \int d^{10} x \sqrt{-g} e^{-2 \Phi} \\
& \times\left(\mathcal{R}+4 \nabla_{M} \Phi \nabla^{M} \Phi-\frac{1}{2}\left|F_{4}\right|^{2}\right),
\end{aligned}
$$

where $2 k_{10}^{2}=16 G_{10} / g_{s}^{2}=(2 \pi)^{7} l_{s}^{8}, G_{10}$ is $10 \mathrm{~d}$ Newton constant and $F_{4}=d C_{3}$ is the field strength of the RamondRamond (R-R) 3-form $C_{3}$ with,

$\left|F_{4}\right|^{2}=\frac{1}{4 !} F_{A B C D} F^{A B C D}$.

The equations of motion could be obtained by varying the action (A.1) with respect to the metric $g_{M N}$, the dilaton field $\Phi$ and the R-R form $C_{3}$ which are,

$$
\begin{aligned}
& \mathcal{R}_{M N}-\frac{1}{2} g_{M N} \mathcal{R}+2 \nabla_{M} \Phi \nabla_{N} \Phi \\
& \quad-\frac{e^{2 \Phi}}{4 !}\left(F_{M}^{L P Q} F_{N L P Q}-3 ! g_{M N}\left|F_{4}\right|^{2}\right)=0, \\
& \mathcal{R}+4 \nabla_{M} \nabla^{M} \Phi-4 \nabla_{M} \Phi \nabla^{M} \Phi=0, \\
& \frac{1}{\sqrt{-g}} \partial_{M}\left[\sqrt{-g} F^{M N P Q}\right]=0 .
\end{aligned}
$$

Note that the solution given in (2.7) exactly satisfies the above equations of motion. The Ramond-Ramond strength $F_{4}$ needs to be supported by $N_{c}$ units of flux on $S^{4}$ which satisfies,

$\frac{k_{10} N_{c}}{\sqrt{\pi} l_{S}}=\int_{S^{4}} F_{4}$.

The factor $N_{c}$ arises because in the WSS model we consider a stack of $N_{c}$ D4-branes.

\section{Appendix B: The action of the D-brane with non-Abelian excitation}

The action of D-branes with non-Abelian excitations describes the dynamics of coincident $N \mathrm{D} p$-branes. It could be obtained by T-duality which is the standard technique in string theory. Let us consider a stack of $\mathrm{D} p$-branes in $D$ dimensional spacetime parametrized by $\left\{X^{\mu}\right\}, \mu=$ $0,1 \ldots D-1$. Then we use the indices $a, b=0,1 \ldots p$ and $i, j, k=p+1 \ldots D-1$ to denote respectively the directions parallel and vertical to the $\mathrm{D} p$-branes in the spacetime. The complete bosonic action of such $\mathrm{D} p$-branes is therefore collected as,

$S_{\mathrm{D}_{p}-\text { branes }}=S_{\mathrm{DBI}}+S_{\mathrm{CS}}$,

where

$$
\begin{aligned}
S_{\mathrm{DBI}}= & -T_{p} \mathrm{~S} \operatorname{Tr} \int d^{p+1} \xi e^{-\Phi} \\
& \times \sqrt{\operatorname{det}\left[Q^{i}{ }_{j}\right] \operatorname{det}\left\{-\left[E_{a b}+E_{a i}\left(Q^{-1}-\delta\right)^{i j} E_{j b}+2 \pi \alpha^{\prime} F_{a b}\right]\right\}}, \\
S_{\mathrm{CS}}= & \mu_{p} \sum_{n=0,1} \int_{\mathrm{D}_{p} \text {-branes }} C_{p-2 n+1} \wedge \frac{\left(B+2 \pi \alpha^{\prime} F\right)^{n}}{n !}, \\
Q^{i}{ }_{j}= & \delta^{i}{ }_{j}+2 \pi \alpha^{\prime}\left[\varphi^{i}, \varphi^{k}\right] E_{k j}, E_{\mu \nu}=g_{\mu \nu}+B_{\mu \nu} .
\end{aligned}
$$

Here $g_{\mu \nu}, B_{\mu \nu}$ is the metric of the $D$ dimensional spacetime and the 2-form field respectively. The "STr" refers to the "symmetric trace" and $F$ is the non-Abelian gauge field strength defined as $F_{a b}=\partial_{[a} A_{b]}+A_{[a} A_{b]}$. The transverse modes of the $\mathrm{D} p$-branes are denoted as $\varphi^{i}$ 's which are in fact the "T-dualitized" coordinates given by $2 \pi \alpha^{\prime} \varphi^{i}=X^{i}$. Note that we have chosen the "static gauge" throughout the manuscript in order to gauge away the 2 -form field $B$ i.e. $B_{\mu \nu}=0$ and defined $\varphi^{i=9} \equiv \Psi$ in (3.14) since there is only one transverse coordinate for the case of $\mathrm{D} 8 / \overline{\mathrm{D} 8}$-branes.

By keeping these in mind, the DBI action in (B.2) could be expanded as,

$$
\begin{gathered}
S_{\mathrm{DBI}}=-T_{p} \operatorname{Tr} \int d^{p+1} \xi e^{-\Phi} \sqrt{-g}\left[1+\frac{1}{4}\left(2 \pi \alpha^{\prime}\right)^{2} F_{a b} F^{a b}\right. \\
\left.+\frac{1}{2} D_{a} \varphi^{i} D^{a} \varphi^{i}+\frac{1}{4}\left[\varphi^{i}, \varphi^{j}\right]^{2}\right]+ \text { high orders. }
\end{gathered}
$$

Note the gauge field $A_{a}$ and scalar field $\varphi^{i}$ 's are all in the adjoint representation of $U(N)$.

As a D-brane is usually supersymmetric, there may be superpartner terms to the actions given in (B.2). Nonetheless we do not attempt to give the explicit action of those fermionic superpartners since they are irrelevant to the calculations in this manuscript. However in the WSS model, one might find it remains to have supersymmetry on the $N_{f}$ flavour branes below the scale of $M_{K K}$ although the $N_{c}$ D4branes are compactified (non-supersymmetric) [46]. Accordingly it forces us to include the superpartners of the mesons 
on the flavour branes. This becomes indeed a theoretical issue of this model because the meson spectrum containing superpartners would be unrealistic. In order to figure out this problem in this model, we are motivated by the mechanisms in the compactification of the $N_{c}$ D4-branes and the processes are as follows. First let us compact the time direction in $10 \mathrm{~d}$ solution (2.7) on a circle with the period/mass scale $\delta t \sim M_{T}^{-1} \gg 1$. Then we require the boundary conditions of the bosonic and the supersymmetrically fermionic fields on D8-brane are periodic and anti-periodic respectively. So the supersymmetric fermions become massive thus decoupled below the scale $M_{T}$. Since the mass scale $M_{T}$ corresponds to the temperature in the dual field theory, it means there is not any superpartners of the mesons below the (very low) temperature $T=M_{T} \ll 1$. Hence the meson spectrum close to zero temperature is non-supersymmetric unless we take the limitation of $\delta t \rightarrow \infty$ i.e. the case of zero-temperature. This scheme provides a non-suppersymmetric meson spectrum at very low but finite temperature and it would be reasonable since zero-temperature case in realistic physics is out of reach thus it could be supersymmetric.

\section{Appendix C: The interactions of glueball and $\pi, \rho$ mesons}

\section{C1: The exotic scalar glueball}

Let identify the lowest modes in (3.6) as vector $\rho$ meson and scalar $\pi$ meson in the sector of the light flavor, then substitute (4.3), (4.4) into the Yang-Mills action in (3.3) and perform the integration over $Z$, it leads to the following $4 \mathrm{~d}$ effective action for the interaction of $\pi, \rho$ meson and the exotic scalar glueball,

$$
\begin{aligned}
& S_{G_{E}}^{\pi-\rho}=-\operatorname{Tr} \int d^{4} x\left\{c _ { 1 } \left[\frac{1}{2} \partial_{\mu} \pi \partial_{\nu} \pi \frac{\partial^{\mu} \partial^{\nu}}{M_{E}^{2}} G_{E}\right.\right. \\
& \left.\quad+\frac{1}{4}\left(\partial_{\mu} \pi\right)^{2}\left(1-\frac{\partial^{2}}{M_{E}^{2}}\right) G_{E}\right] \\
& +c_{2} M_{K K}^{2}\left[\frac{1}{2} \rho_{\mu} \rho_{\nu} \frac{\partial^{\mu} \partial^{\nu}}{M_{E}^{2}} G_{E}+\frac{1}{4}\left(\rho_{\mu}\right)^{2}\left(1-\frac{\partial^{2}}{M_{E}^{2}}\right) G_{E}\right] \\
& +c_{3}\left[\frac{1}{2} \eta^{\sigma \lambda} \partial_{[\mu} \rho_{\sigma]} \partial \partial_{\nu} \rho_{\lambda]} \frac{\partial^{\mu} \partial^{\nu}}{M_{E}^{2}} G_{E}\right. \\
& \left.\quad-\frac{1}{8} \partial_{[\mu} \rho_{\nu]} \partial^{[\mu} \rho^{\nu]}\left(1+\frac{\partial^{2}}{M_{E}^{2}}\right) G_{E}\right] \\
& +c_{4} \frac{3}{2 M_{E}^{2}} \rho_{\mu} \partial^{[\mu} \rho^{\nu]} \partial_{\nu} G_{E} \\
& +c_{5}\left[\partial_{\mu} \pi\left[\pi, \rho_{\nu}\right] \frac{\partial^{\mu} \partial^{\nu}}{M_{E}^{2}} G_{E}+\frac{1}{2} \partial_{\mu} \pi\left[\pi, \rho^{\mu}\right]\left(1-\frac{\partial^{2}}{M_{E}^{2}}\right) G_{E}\right] \\
& +\left[\frac{1}{2} \tilde{c}_{1}\left(\partial_{\mu} \pi\right)^{2}+\frac{1}{2} \tilde{c}_{2} M_{K K}^{2}\left(\rho_{\mu}\right)^{2}+\frac{1}{4} \tilde{c}_{3} \partial\left[\mu \rho_{\nu]} \partial^{[\mu} \rho^{\nu]}\right.\right. \\
& \left.\left.+\tilde{c}_{5} \partial_{\mu} \pi\left[\pi, \rho^{\mu}\right]\right] G_{E}\right\},
\end{aligned}
$$

where the coefficients $c_{i}$ 's and $\tilde{c}_{i}$ 's are,

$$
\begin{aligned}
c_{1} & =\int d Z \frac{\bar{H}_{E}}{\pi K}, \quad c_{2}=2 \kappa \int d Z K\left(\psi_{1}^{\prime}\right) \bar{H}_{E}, \\
c_{3} & =2 \kappa \int d Z K^{-1 / 3}\left(\psi_{1}\right)^{2} \bar{H}_{E}, \\
c_{4} & =2 \kappa M_{K K}^{2} \int d Z \frac{20 Z K}{(5 K-2)^{2}} \psi_{1} \psi_{1}^{\prime} H_{E}, \\
c_{5} & =\int d Z \frac{\psi_{1} \bar{H}_{E}}{\pi K}, \quad \tilde{c}_{1}=\int d Z \frac{H_{E}}{4 \pi K} \\
\tilde{c}_{2} & =\frac{1}{2} \kappa \int d Z K\left(\psi_{1}^{\prime}\right)^{2} H_{E}, \\
\tilde{c}_{3} & =\frac{1}{2} \kappa \int d Z K^{-1 / 3}\left(\psi_{1}\right)^{2} H_{E}, \\
\tilde{c}_{5} & =\int d Z \frac{\psi_{1} H_{E}}{4 \pi K} .
\end{aligned}
$$

$\mathrm{C} 2$ : The dilatonic and tensor glueball

The action for the interaction of dilatonic glueball and $\pi, \rho$ mesons could be obtained by plugging the (2.14) into action (3.3) then performing the integration over $Z$. We collect the resultantly quadratic terms in $\pi, \rho$ as,

$$
\begin{aligned}
S_{G_{D}}^{\pi-\rho}= & \operatorname{Tr} \int d^{4} x\left\{\frac{1}{2} d_{1} \partial_{\mu} \pi \partial_{\nu} \pi\left(\eta^{\mu \nu}-\frac{\partial^{\mu} \partial^{\nu}}{M_{D}^{2}}\right) G_{D}\right. \\
& +\frac{1}{2} d_{2} M_{K K}^{2} \rho_{\mu} \rho_{\nu}\left(\eta^{\mu \nu}-\frac{\partial^{\mu} \partial^{\nu}}{M_{D}^{2}}\right) G_{D} \\
& +\frac{1}{2} d_{3} \eta^{\lambda \sigma} \partial_{[\mu} \rho_{\sigma]} \partial_{[\nu} \rho_{\lambda]}\left(\eta^{\mu \nu}-\frac{\partial^{\mu} \partial^{\nu}}{M_{D}^{2}}\right) G_{D} \\
& \left.+d_{5} \partial_{\mu} \pi\left[\pi, \rho_{\nu}\right]\left(\eta^{\mu \nu}-\frac{\partial^{\mu} \partial^{\nu}}{M_{D}^{2}}\right) G_{D}\right\},
\end{aligned}
$$

where the coefficients are,

$$
\begin{aligned}
& d_{1}=\int d Z \frac{H_{D}}{\pi K}, d_{2}=2 \kappa \int d Z K\left(\psi_{1}^{\prime}\right)^{2} H_{D}, \\
& d_{3}=2 \kappa \int d Z K^{-1 / 3}\left(\psi_{1}\right)^{2} H_{D}, d_{5}=\int d Z \frac{\psi_{1} H_{D}}{\pi K} .
\end{aligned}
$$

And similarly, the interaction action of tensor glueball and $\pi, \rho$ mesons is given as,

$$
\begin{aligned}
S_{G_{T}}^{\pi-\rho}= & \operatorname{Tr} \int d^{4} x\left\{\frac{1}{2} t_{1} T^{\mu \nu} \partial_{\mu} \pi \partial_{\nu} \pi+\frac{1}{2} t_{2} M_{K K}^{2} T^{\mu \nu} \rho_{\mu} \rho_{\nu}\right. \\
& \left.+\frac{1}{2} t_{3} T^{\mu \nu} \eta^{\lambda \sigma} \partial_{[\mu} \rho_{\sigma]} \partial_{[v} \rho_{\lambda]}+t_{5} \partial_{\mu} \pi\left[\pi, \rho_{\nu}\right] T^{\mu \nu}\right\},
\end{aligned}
$$

with the coefficients $t_{i}=\sqrt{6} d_{i}$. 
Appendix D: The renormalization of the quadric D8brane action

In the holographic approach, the full action of the D-brane usually contains a counterterm [40]. While the holographic counterterm for the probe D8-brane in this model is less clear, it can be constructed by analyzing its asymptotics and computed as $[17,21,41](\epsilon \rightarrow 0)$,

$$
\begin{aligned}
S_{c . t}^{D 8}= & \frac{Q_{f}}{2 k_{10}^{2} l_{s}^{2}} \int d^{9} x \frac{\sqrt{h}}{\sqrt{h_{44}}}\left[\chi_{1} \frac{\mathcal{R}}{g_{s}^{1 / 3}} e^{-2 \phi / 3}-2 \chi_{2} \frac{\mathcal{R}^{2}}{g_{s}^{2 / 3}} e^{-\phi / 3}\right. \\
& \left.\times\left(K-\frac{8}{3} n \cdot \nabla \phi-n \cdot \frac{\nabla\left(\sqrt{g_{44}}\right)}{\sqrt{g_{44}}}\right)\right] \\
= & a \mathcal{V} \epsilon_{f}\left[\frac{\chi_{1}-4 \chi_{2}}{3(3)^{1 / 6} \epsilon^{7 / 6}}+\frac{7 \chi_{1}-4 \chi_{2}}{12(3)^{1 / 6} \epsilon^{1 / 6}}\right] .
\end{aligned}
$$

where

$$
\begin{aligned}
Q_{f} & =\frac{2 k_{10}^{2} N_{f} T_{8} M_{K K} l_{s}^{2}}{\pi}, \\
a \mathcal{V} & =\frac{1}{2 k_{0}^{2} g_{s}^{2}} \frac{V_{3}}{T} \frac{2 \pi}{M_{K K}} V_{S^{4}} U_{K K}^{3}, \\
\epsilon_{f} & =\frac{R^{3 / 2} U_{K K}^{1 / 2} g_{s}}{l_{s}^{2}} Q_{f} .
\end{aligned}
$$

And $\chi_{1,2}$ are two parameters, $h$ is the determinant of the metric at the UV boundary, $K$ is the trace of the boundary extrinsic curvature whose explicit form is

$K=\left.h^{M N} \nabla_{M} n_{N}\right|_{\text {boundary }}, \quad n^{M}=-\frac{\delta^{M U}}{\sqrt{g_{U U}}}$.

If $\chi_{1}=\frac{16}{7}, \chi_{2}=\frac{1}{2}$, this counterterm exactly cancels the divergence in the DBI action of the probe D8-branes,

$$
\begin{aligned}
S_{\mathrm{DBI}}^{D 8} & =\frac{Q_{f}}{2 k_{10}^{2}} \frac{2 \pi}{M_{K K}} \int d^{9} x e^{-\phi} \sqrt{\operatorname{det} g_{9}}=a \mathcal{V} \epsilon_{f} d_{1}, \\
d_{1} & =\frac{2}{21(3)^{1 / 6} \epsilon^{7 / 6}}+\frac{7}{6(3)^{1 / 6} \epsilon^{1 / 6}}+\frac{24}{7} \frac{\pi^{3 / 2}}{\Gamma\left(-\frac{2}{3}\right) \Gamma\left(\frac{1}{6}\right)} .
\end{aligned}
$$

So taking into account the quadric action of the probe brane, the counterterm including the transverse modes can be further constructed by using (D.1) or equivalently (D.4) which leads to an additional counterterm to (3.14) as

$$
S_{c . t .}^{\Psi}=\frac{1}{4} \tilde{T}_{8} \operatorname{Tr} \int d^{9} x e^{-\Phi} \sqrt{-\operatorname{det} g_{9}}[\Psi, \Psi]^{2} .
$$

Following the discussion from (3.15) to (3.16), this term can be written as

$$
S_{c . t}^{\Psi}=\left.\left[v^{2} \frac{\left(N_{f}+1\right)^{2}}{N_{f}^{2}} d_{1} \int d Z g^{M N} \Phi_{M}^{\dagger} \Phi_{N}\right]\right|_{\text {boundary }}
$$

$$
\rightarrow \begin{cases}M_{S, n}^{2}\left(Z_{H} \rightarrow \infty\right) Q_{n}^{\dagger}(x) Q_{n}(x), & \text { for scalar } \\ M_{V, n}^{2}\left(Z_{H} \rightarrow \infty\right) Q_{\mu, n}^{\dagger}(x) Q_{, n}^{\mu}(x), & \text { for vector }\end{cases}
$$

where

$$
\begin{aligned}
& M_{V, n}^{2}\left(Z_{H}\right)=v_{V}^{2} \kappa \int_{-Z_{H}}^{+Z_{H}} d Z K(Z)^{1 / 6} \phi_{n}(Z) \phi_{n}(Z), \\
& M_{S, n}^{2}\left(Z_{H}\right)=v_{S}^{2} \kappa \int_{-Z_{H}}^{+Z_{H}} d Z K(Z)^{3 / 2} \tilde{\phi}_{n}(Z) \tilde{\phi}_{n}(Z) .
\end{aligned}
$$

Hence the potential infinities in $M_{V}$ and $M_{S}$ would depend on the behaviors of the basis functions $\left\{\phi_{m}, \tilde{\phi}_{m}\right\}$ at large $Z$ and can be removed by (D.6). Then the renormalized mass (3.21), (3.25) should be given as

$$
\begin{aligned}
& M_{V, \text { ren }}^{2}=v_{V}^{2} \kappa \int_{-\infty}^{+\infty} d Z K(Z)^{1 / 6} \phi_{n}(Z) \phi_{n}(Z)-S_{c . t}^{\Psi}, \\
& M_{S, \text { ren }}^{2}=v_{S}^{2} \kappa \int_{-\infty}^{+\infty} d Z K(Z)^{3 / 2} \tilde{\phi}_{n}(Z) \tilde{\phi}_{n}(Z)-S_{c . t}^{\Psi} .
\end{aligned}
$$

As an effective theory of meson, the mass term presented in (3.20) should be interpreted as the renormalized mass. And it implies this setup is valid below the heavy-flavoured energy scale. Finally since the basis function may not be analytical, let us numerically evaluate $S_{c . t}^{\Psi}$ for the lowest scalar and vector modes presented in this manuscript (in $Z$ coordinate),

$$
\begin{aligned}
S_{\text {c.t }}^{\text {Vector }} & =\left.\left[v^{2} \frac{\left(N_{f}+1\right)^{2}}{N_{f}^{2}} d_{1} \int d Z g^{M N} \Phi_{M}^{\dagger} \Phi_{N}\right]\right|_{\text {boundary }} \\
& \left.\simeq 0.159155 M_{K K}^{-2} U_{K K}^{-2} v_{V}^{2} \kappa \log \varepsilon\right|_{\varepsilon \rightarrow 0} \\
S_{\text {c.t }}^{\text {Scalar }} & =\left.\left[v^{2} \frac{\left(N_{f}+1\right)^{2}}{N_{f}^{2}} d_{1} \int d Z g^{M N} \Phi_{M}^{\dagger} \Phi_{N}\right]\right|_{\text {boundary }} \\
& \simeq 10^{-15} M_{K K}^{-2} U_{K K}^{-2} v_{S}^{2} \kappa \simeq 0 .
\end{aligned}
$$

Then using (D.8) it gives a finite numerical result

$M_{V, \text { ren }}^{2} \simeq 1.386 M_{K K}^{-2} U_{K K}^{-2} v_{V}^{2} \kappa^{2}$,

$M_{S, \text { ren }}^{2} \simeq 1.237 M_{K K}^{-2} U_{K K}^{-2} v_{V}^{2} \kappa^{2}$.

According to the expirenmental data about the D-meson, fitting $M_{S} \simeq 1754 \mathrm{MeV}, M_{V} \simeq 2007 \mathrm{MeV}$, it leads to (5.4) for the numerical calculation in this paper.

\section{References}

1. H. Fritzsch, M. Gell-Mann, Current algebra: quarks and what else? eConf C720906V2, 135-165 (1972). arXiv:hep-ph/0208010 
2. H. Fritzsch, P. Minkowski, $\Psi$ resonances, gluons and the Zweig rule. Nuovo Cim. A 30, 393 (1975)

3. R. Jaffe, K. Johnson, Unconventional states of confined quarks and gluons. Phys. Lett. B 60, 201 (1976)

4. C.J. Morningstar, M.J. Peardon, The Glueball spectrum from an anisotropic lattice study. Phys. Rev. D 60, 034509 (1999). arXiv:hep-lat/9901004

5. Y. Chen, A. Alexandru, S. Dong, T. Draper, I. Horvath et al., Glueball spectrum and matrix elements on anisotropic lattices. Phys. Rev. D 73, 014516 (2006). arXiv:hep-lat/0510074

6. M. Loan, X.-Q. Luo, Z.-H. Luo, Monte Carlo study of glueball masses in the Hamiltonian limit of SU(3) lattice gauge theory. Int. J. Mod. Phys. A 21, 2905-2936 (2006). arXiv:hep-lat/0503038

7. W.J. Lee, D. Weingarten, Scalar quarkonium masses and mixing with the lightest scalar glueball. Phys. Rev. D 61, 014015 (2000). arXiv:hep-lat/9910008

8. J.M. Maldacena, The large $\mathrm{N}$ limit of superconformal field theories and supergravity. Int. J. Theor. Phys. 38, 1113-1133 (1999). arXiv:hep-th/9711200

9. O. Aharony, S.S. Gubser, J.M. Maldacena, H. Ooguri, Y. Oz, Large $\mathrm{N}$ field theories, string theory and gravity. Phys. Rep. 323, 183-386 (2000). arXiv:hep-th/9905111

10. E. Witten, Anti-de Sitter space and holography. Adv. Theor. Math. Phys. 2, 253 (1998). arXiv:hep-th/9802150

11. E. Witten, Anti-de Sitter space, thermal phase transition, and confinement in gauge theories. Adv. Theor. Math. Phys. 2, 505-532 (1998). arXiv:hep-th/9803131

12. T. Sakai, S. Sugimoto, Low energy hadron physics in holographic QCD. Prog. Theor. Phys. 113, 843-882 (2005). arXiv:hep-th/0412141

13. T. Sakai, S. Sugimoto, More on a holographic dual of QCD. Prog. Theor. Phys. 114, 1083-1118 (2005). arXiv:hep-th/0507073

14. A. Rebhan, The Witten-Sakai-Sugimoto model: a brief review and some recent results. EPJ Web Conf. 95, 02005 (2015). arXiv: 1410.8858

15. O. Bergman, Gilad Lifschytz, M. Lippert, Holographic nuclear physics. JHEP 0711, 056 (2007). arXiv:0708.0326

16. S. Li, A. Schmitt, Q. Wang, From holography towards realworld nuclear matter. Phys. Rev. D 92(2), 026006 (2015). arXiv:1505.04886

17. F. Bigazzi, A.L. Cotrone, Holographic QCD with dynamical flavors. JHEP 1501, 104 (2015). arXiv: 1410.2443

18. M. Rozali, H.-H. Shieh, M. Van Raamsdonk, W. Jackson, Cold nuclear matter in holographic QCD. JHEP 0801, 053 (2008). arXiv:0708.1322

19. A. Rebhan, A. Schmitt, S.A. Stricker, Meson supercurrents and the Meissner effect in the Sakai-Sugimoto model. JHEP 0905, 084 (2009). arXiv:0811.3533

20. H. Hata, Tadakatsu Sakai, S. Sugimoto, S. Yamato, Baryons from instantons in holographic QCD. Prog. Theor. Phys. 117, 1157 (2007). arXiv:hep-th/0701280

21. S. Li, T. Jia, Dynamically flavored description of holographic QCD in the presence of a magnetic field. Phys. Rev. D 96(6), 066032 (2017). arXiv: 1604.07197

22. Edward Witten, Baryons and branes in anti-de Sitter space. JHEP 9807, 006 (1998). arXiv:hep-th/9805112

23. N.R. Constable, R.C. Myers, Spin-two glueballs, positive energy theorems and the AdS/CFT correspondence. JHEP 9910, 037 (1999). arXiv:hep-th/9908175
24. R.C. Brower, S.D. Mathur, C.-I. Tan, Glueball spectrum for QCD from AdS supergravity duality. Nucl. Phys. B 587, 249-276 (2000). arXiv:hep-th/0003115

25. K. Hashimoto, Chung-I Tan, S. Terashima, Glueball decay in holographic QCD. Phys. Rev. D 77, 086001 (2008). arXiv:0709.2208

26. F. Brünner, D. Parganlija, A. Rebhan, Glueball decay rates in the Witten-Sakai-Sugimoto model. Phys. Rev. D 91(10), 106002 (2015). [Erratum: Phys. Rev. D 93(10), 109903 (2016)]. arXiv: 1501.07906

27. S. Li, Glueball-baryon interactions in holographic QCD. Phys. Lett. B 773, 142-149 (2017). arXiv:1509.06914

28. Y. Liu, I. Zahed, Holographic heavy-light chiral effective action. Phys. Rev. D 95, 056022 (2016). arXiv:1611.03757

29. Y. Liu, I. Zahed, Heavy-light mesons in chiral AdS/QCD. Phys. Lett. B. arXiv:1611.04400

30. K. Becker, M. Becker, J.H. Schwarz, String Theory and M-Theory, A Modern Introduction (Cambridge University Press, Cambridge, 2007)

31. R.C. Myers, Dielectric-branes. JHEP 9912, 022 (1999). arXiv:hep-th/9910053

32. BESIII Collaboration, Measurement of yCP in $D^{0}-\bar{D}^{0}$ oscillation using quantum correlations in $e^{-} e^{+} \rightarrow D^{0} \bar{D}^{0}$ at $\sqrt{s}=3.773 \mathrm{GeV}$. Phys. Lett. B 744, 339-346 (2015). arXiv:1501.01378

33. CDF Collaboration, Observation of Bs-Bsbar oscillations. Phys. Rev. Lett. 97, 242003 (2006). arXiv:hep-ex/0609040

34. S. Li, Holographic description of heavy-flavored baryonic matter decay involving glueball. Phys. Rev. D 99(4), 046013 (2019). arXiv: 1812.03482

35. E. Witten, String theory dynamics in various dimensions. Nucl. Phys. B 443, 85-126 (1995). arXiv:hep-th/9503124

36. S. Sugimoto, K. Takahashi, QED and string theory. JHEP 0404, 051 (2004). arXiv:hep-th/0403247

37. S. Li, Holographic heavy-baryons in the Witten-Sakai-Sugimoto model with the D0-D4 background. Phys. Rev. D 96(10), 106018 (2017). arXiv:1707.06439

38. W. Cai, S. Li, Holographic three flavor baryon in the Witten-SakaiSugimoto model with the D0-D4 background. Eur. Phys. J. C 78(6), 446 (2018). arXiv: 1712.06304

39. Y. Liu, I. Zahed, Heavy baryons and their exotics from instantons in holographic QCD. Phys. Rev. D 95(11), 116012 (2017). arXiv: 1704.03412

40. A. Karch, A. O'Bannon, K. Skenderis, Holographic renormalization of probe D-branes in AdS/CFT. JHEP 06(04), 015 (2006). arXiv:hep-th/0512125

41. P. Benincasa, A note on holographic renormalization of probe Dbranes. arXiv:0903.4356

42. Y.K. Hsiao, C.Q. Geng, Identifying glueball at $3.02 \mathrm{GeV}$ in baryonic B decays. Phys. Lett. B 727, 168-171 (2013). arXiv:1302.3331

43. C. Wu, Z. Xiao, D. Zhou, Sakai-Sugimoto model in D0-D4 background. Phys. Rev. D 88(2), 026016 (2013). arXiv:1304.2111

44. S. Seki, S.-J. Sin, A new model of holographic QCD and chiral condensate in dense matter. JHEP 1310, 223 (2013). arXiv:1304.7097

45. S. Li, T. Jia, Matrix model and holographic baryons in the D0-D4 background. Phys. Rev. D 92(4), 046007 (2015). arXiv: 1506.00068

46. R. Heise, H.G. Svendsen, A Note on fermions in holographic QCD. JHEP 0708, 065 (2007). arXiv:0706.2253 